\title{
Non-Newtonian Nanofluid in a Micro Planar Sudden Expansion Considering Variable Properties
}

Meng-He Sun ${ }^{\text {a }}$, Xin-Rong Zhang, ${ }^{\text {a,b,* }}$

\section{Abstract}

The heat transfer and flow pattern of $\mathrm{Al}_{2} \mathrm{O}_{3}$-water nanofluid in a micro planar sudden expansion with constant heat flux boundary conditions are numerically studied. The $\mathrm{Al}_{2} \mathrm{O}_{3}$-water nanofluid is treated as a single phase non-Newtonian fluid with variable physical properties and the power-law rheology is adopted to describe the characteristics of the flow, in which the flow behavior index depend on the nanoparticle volume fraction. A

$1 / 35$ 
systematic study of the $\mathrm{Al}_{2} \mathrm{O}_{3}$-water nanofluid for a wide range of generalized Reynolds number, $50 \leq R e_{\text {gen }} \leq 500$, and nanoparticle volume fraction $0 \% \leq \varphi \leq 3 \%$ is presented. The critical generalized Reynolds number at which flow bifurcation occurs is carefully studied and the heat transfer enhancement due to the non-Newtonian rheology is reported. It is shown that the flow bifurcation is delayed when the nanoparticle volume fraction increases and is advanced as the boundary heat transfer rate increases. The heat transfer deterioration brought by the recirculation area is reduced under a higher nanoparticle volume fraction and the reduction ratio increases with the generalized Reynolds number. The non-Newtonian model results are also compared with the results using a Newtonian model, which indicates that there will be a huge overestimate on the system pressure drop if Newtonian model is taken.

\section{Keywords}

Nanofluid, Non-Newtonian, Planar Sudden Expansion, Variable Property 


\section{Introduction}

Nanofluid is a dilute suspension of solid nanometer-size particles and fibers dispersed in a conventional fluid (i.e., water, oil etc.).These particles can be metallic or nonmetallic, such as $\mathrm{Al}_{2} \mathrm{O}_{3}, \mathrm{SiO}_{2}, \mathrm{Cu}, \mathrm{CuO}, \mathrm{ZnO}$ and $\mathrm{TiO}_{2}$ [1]. Nanofluids have been found to possess enhanced thermophysical properties such as thermal conductivity, thermal diffusivity, viscosity and convective heat transfer coefficients compared to those of base fluids like oil or water [2-4]. Researchers reported that nanofluids can clearly exhibit enhanced thermal conductivity, which goes up with increasing nanoparticle volume fraction. Nanofluids often appear better heat transfer performance because of the high thermal conductivity, thus they are widely used to improve the system efficiency. In order to use nanofluids for small scale cooling techniques such as integrated circuits and micro-electro mechanical systems, the investigation on flow and heat transfer of nanofluids in microstructures like microtubes and microchannels is imperatively needed. The system geometries include micro tubes [5], microchannels [6-8] and microchannels of a sudden change (i.e. backward-facing steps [9] and forward-facing steps [10]).

The separation in the fluid flow can be generated from a sudden change in flow geometry. The planar sudden expansion plays an important role in the design of many engineering applications where heating or cooling is required. When a Newtonian fluid flows at low to moderate Reynolds number in a planar channel and encounters a sudden expansion, flow separation occurs resulting in a pair of symmetric recirculating eddies along the downstream walls. When Reynolds number is increased above the critical value, the vortexes become asymmetric, and gradually a third eddy is formed downstream of the smallest of the two main vortexes [11]. The bifurcation phenomenon, consisting of a transition from the symmetric to the asymmetric flow, has great impact on the flow and heat transfer performance of the system and thus is of great interests.

In many realistic situations, the fluids flowing through the devices are non-Newtonian and show complex rheological behavior. A non-Newtonian fluid is one whose flow curve (shear stress versus shear rate) is non-linear 
or does not pass through the origin, i.e. where the apparent viscosity (shear stress divided by shear rate) is not constant at a given temperature and pressure but is dependent on flow conditions such as flow geometry, shear rate, etc. and sometimes even on the kinematic history of the fluid element under consideration. Specifically, they can exhibit shear-thinning or shear-thickening viscosity depending on the type of fluid.

In most related works, nanofluids are treated as homogeneous single-phase fluids (with the assumption that the nanoparticles are uniformly distributed in base fluids). Thus, the most common way is to use the macroscopic results from the numerous existed studies on the flow and heat transfer of fluids in large scale structures. Kherbeetet. al [10] used Newtonian model to investigate the flow and heat transfer performance of $\mathrm{Al}_{2} \mathrm{O}_{3}$-water nanofluids in a microchannel of a forward-facing step and set experiment for validation. Although the experimental and numerical results are generally in good agreement, there still exists a gap between the pressure drop obtained from the experiment and that of the simulation. These results indicate that the macroscopic regulations may not be simply extended to apply for microscopic problems due to the appearance of some special phenomena with the shrinkage of characteristic length, e.g. the fluid rheology. Some scholars used two-phase model to numerically simulate the flow and heat transfer performance of nanofluid. Sheikholeslami. et. al [12] used Fourth-order RungeKutta method to analyze thermal radiation on magnetohydrodynamic nanofluid. Eslamian [13] clarified the role of thermophoresis of Rayleigh-Benard laminar natural convection by using a two-phase Lattice Boltzmann Method. In these studies, the convectional fluid is treated as a continuous phase and the nanoparticle is presented by its volume friction. The Brownian motion and thermophoresis effects can be studied in this way, but the fluid rheological behavior is still not considered.

Rheological behaviour of nanofluids affects pressure drop of nanofluids. Additionally it gives an idea of nanoparticle structuring, which can be helpful in predicting the thermal conductivity of nanofluids. One simplified way as has already been used in many works [14-16] is to treat nanofluids as a Newtonian fluid with modified 
physical properties. However, some experimental results have shown that the Newtonian model may not be accurate enough for describing the behavior of some particular nanofluids. Some researchers observed a shear-thinning fluid behavior [17] and some reported that the increasing nanoparticle volume fraction enhance the shear-thinning behavior [18]. The shear-thinning behavior has been found in different species of nanofluids, including $\mathrm{Cu}$-water [17, 19], carbon nanotube-water [18], $\mathrm{Al}_{2} \mathrm{O}_{3}$-water [20-22] and $\mathrm{TiO}_{2}$-water [23-25]. Although different kinds of nanofluids show non-Newtonian behavior, it has to be emphasized that there are still many Newtonian nanofluids. All the suspensions containing $\mathrm{SiO}_{2}$ nanoparticles show Newtonian behaviour [26, 27]. $\mathrm{Al}_{2} \mathrm{O}_{3}$-water nanofluid show non-Newtonian behavior while $\mathrm{Al}_{2} \mathrm{O}_{3}$-EG and $\mathrm{Al}_{2} \mathrm{O}_{3}-\mathrm{PG}$ behave as Newtonian fluid $[28,29]$.Water-based nanofluid containing micro-sized $\mathrm{Al}_{2} \mathrm{O}_{3}$ particle exhibits shear thinning behavior. Tseng and $\mathrm{Wu}$ [21] investigated $\mathrm{Al}_{2} \mathrm{O}_{3}$-water nanofluids with the particle diameter $d=37 \mathrm{~nm}$ and volumetric solid concentrations from $1 \%$ to $16 \%$, and concluded that the suspension generally showed a transition from shear-thinning to shear-thickening as the shear rate exceeded a certain critical. Also, this critical value of shear rate increased with the rise of nanoparticle concentration.

Some scholars have done remarkable works on the numerical simulation of non-Newtonian nanofluids. J. Niu et al. [5] theoretically studied the slip-flow and heat transfer of a non-Newtonian nanofluid in a microtube, and concluded that the heat transfer rate of the nanofluid in the microtube can be enhanced due to the non-Newtonian rheology and slip boundary effects. GH.R. Kefayati [30] used Finite Difference Lattice Boltzmann Method (FDLBM) to study the heat transfer and entropy generation on laminar natural convection of non-Newtonian $\mathrm{Cu}$-water nanofluids in the presence of an external horizontal magnetic field in a square cavity. Results indicate that the augmentation of the power-law index causes heat transfer to drop in the absence of the magnetic field, by contrast, the heat transfer increases with the rise of power-law index in the presence of the magnetic field. Li et. al. [31] used power-law model to numerically simulate laminar forced convection nanofluids in a horizontal parallel 
plate. Ellahi et. al. [32] used the homotopy analysis method (HAM) to get the analytical sultions of non-Newtonian nanofluids with Reynolds' model and Vogel's model. Later this method were used to gave the analytical solutions of the magnetohydrodynamic (MHD) flow of non-Newtonian nanofluid in a pipe [33] and nanofluid flow through composite stenosed arteries with permeable walls [34]. Some recent researches have been focused on different types of non-Newtonian nanofluid such as Jeffrey nanofluid [35] and Oldroyd-B nanofluid [36].

The objective of this work is to investigate the heat transfer and flow pattern of $\mathrm{Al}_{2} \mathrm{O}_{3}$-water non-Newtonian nanofluid in a micro planar sudden expansion. $\mathrm{Al}_{2} \mathrm{O}_{3}$-water nanofluid is chosen because it has identifiable non-Newtonian behavior and a rich set of experimental data. According to the author's knowledge, the flow bifurcation and heat transfer enhancement of the specific model is still lacking. In the present study, we present a systematic study of the $\mathrm{Al}_{2} \mathrm{O}_{3}$-water nanofluid for a wide range of generalized Reynolds number, $50 \leq R e_{\text {gen }} \leq 500$, and nanoparticle volume fraction $0 \% \leq \varphi \leq 3 \%$. The boundary conditions are set to be different values of constant heat flux rate. The symmetry breaking flow bifurcation is carefully studied and the heat transfer enhancement due to the non-Newtonian rheology is reported. We also compare the non-Newtonian model results with the results using a Newtonian model.

\section{Mathematical Models}

\section{Problem description}

Laminar convection flows in the planar sudden expansion are numerically simulated. The problem under study is illustrated schematically in Fig. 1a, and the nomenclature used to refer to the various characteristic lengths of the vortices is illustrated in Fig. 1b. A two-dimensional, planar channel of width $h$ has a sudden expansion to a second channel of width $H$. The center of the co-ordinate axes system lies at the bottom of the geometry expansion plane. The upstream channel has a length $L_{a}=50 h$ and that of the downstream channel is $L_{b}=200 h$. The both side of the 
downstream boundary wall has a constant heat flux of $q_{w}$. The inlet is located far enough to ensure that the inlet fluid flow be fully developed before the expansion. Similarly, the outlet is located far away from the region where the separating flow regions occur.

The upstream width of the duct $(h)$ is $100 \mu \mathrm{m}$; its downstream width $(H)$ is $300 \mu \mathrm{m}$, and the expansion ratio is $E R=H / h=3$. Cold nanofluid of temperature $T_{0}$ flows from the left to the right. The nanofluid is a mixture of water and solid aluminum oxide particles of $30 \mathrm{~nm}$ diameter. The nanoparticles are of uniform shape and size. The nanofluid is incompressible and the flow is laminar. Also the nanofluid is treated as homogeneous single-phase fluids (with the assumption that the nanoparticles are uniformly distributed in base fluids; the liquid and solid are in thermal equilibrium). The thermophysical properties of the nanofluid are assumed to be temperature dependent.

The effect of buoyancy is neglected, since it is not significant in comparison with the microchannel flow.

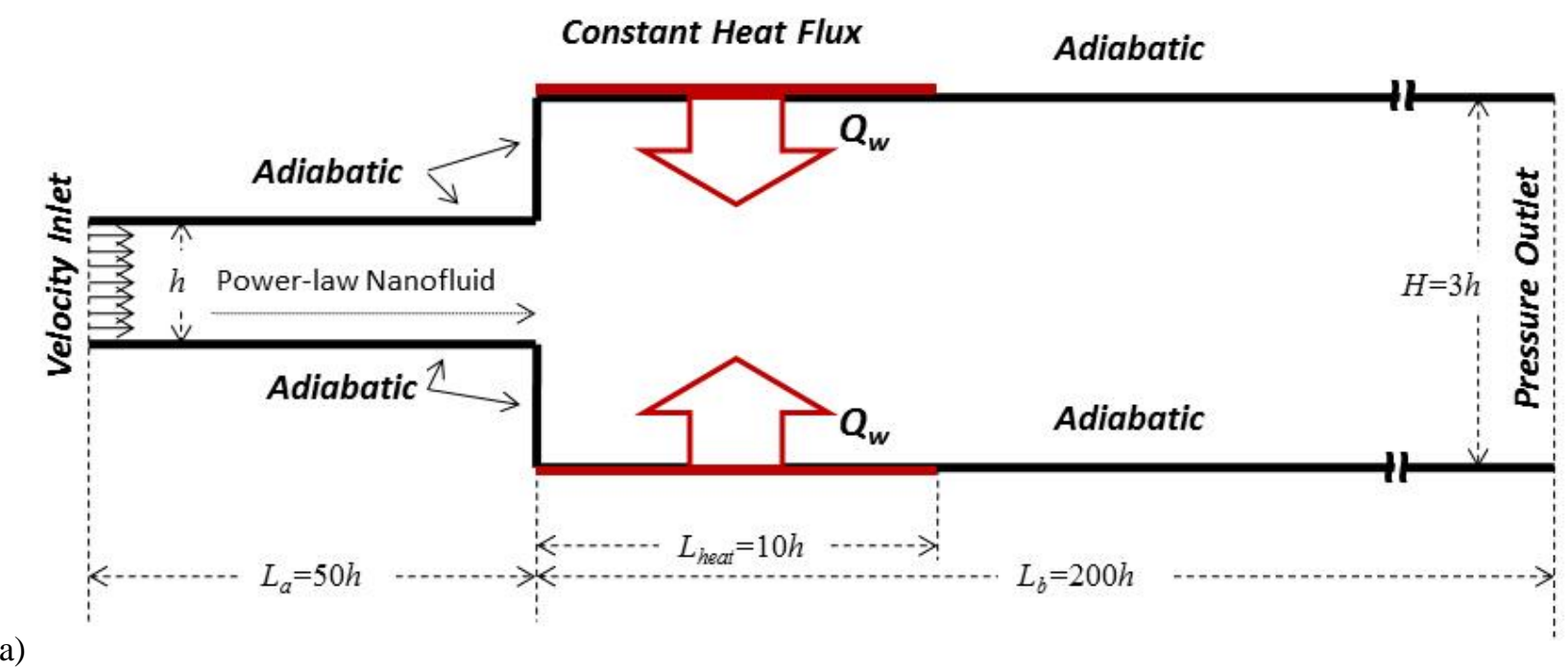




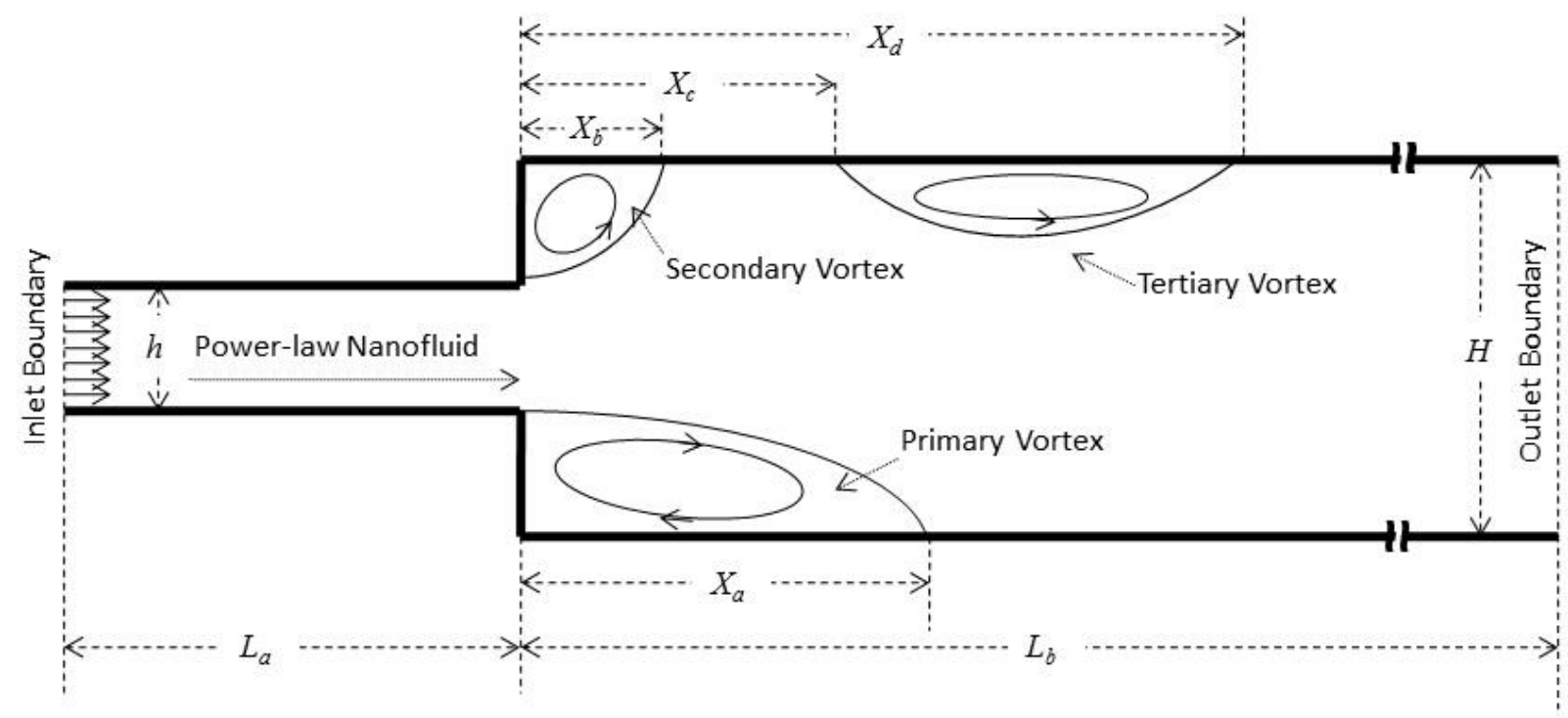

(b)

Fig 1 Illustration of (a) the physical model of two-dimensional 1:3 sudden planar expansion geometry in the study and (b) the nomenclature used to refer to the various characteristic lengths of the vortices

\section{Thermophysical properties of nanofluid}

In the current study, the nanofluid is treated as single phase fluid. The effective density at reference temperature is [37]

$$
\rho_{n f}=(1-\phi) \rho_{f}+\phi \rho_{p}
$$

The heat capacitance of nanofluid is

$$
(\rho C p)_{n f}=(1-\phi)(\rho C p)_{f}+\phi(\rho C p)_{p}
$$

The effective thermal conductivity of fluid has been determined by the model proposed by Patel et al. [38].

For the two-component entity of spherical-particle suspension the model gives

$$
\frac{k_{e f f}}{k_{f}}=1+(1+c P e) \frac{k_{p} A_{p}}{K_{f} A_{f}}
$$

where

$$
\frac{A_{p}}{A_{f}}=\frac{d_{p}}{d_{f}} \frac{\phi}{(1-\phi)}
$$




$$
P e=\frac{u_{p} d_{p}}{\alpha_{f}}
$$

where $u_{p}$ is the Brownian motion velocity of the particles which is given by

$$
u_{p}=\frac{2 k_{b} T}{\pi \mu_{f} d_{p}^{2}}
$$

where $k_{b}$ is the Boltzmann constant.

The effective dynamic viscosity of the nanofluid as given by Brinkman [39] is as follows:

$$
\mu_{n f}=\frac{\mu_{f}}{(1-\phi)^{2.5}}
$$

The properties of base fluid, water at different temperatures are available in ASHRAE. These properties as a function of temperature were curve fitted. Then they were substituted in the density, specific heat, thermal conductivity and viscosity equations to evaluate the properties of nanofluid at different temperature and concentrations. Therefore, the properties of nanofluids are temperature dependent in the current study. The thermophysical properties of of pure water and $\mathrm{Al}_{2} \mathrm{O}_{3}$ at $T=300 \mathrm{~K}$ are summarized in Table 1 . The curve fitting of the thermophysical properties of nanofluid as a function of temperature are shown in Fig 2.

Table 1Therrmophysical properties of pure water and $\mathrm{Al}_{2} \mathrm{O}_{3}$ at $T=300 \mathrm{~K}$

\begin{tabular}{|l|l|l|}
\hline Thermophysical properties & Water & $\mathrm{Al}_{2} \mathrm{O}_{3}$ \\
\hline Density, $\rho\left(\mathrm{kg} / \mathrm{m}^{3}\right)$ & 998.203 & 3970 \\
\hline Dynamic viscosity, $\mu\left(\mathrm{Ns} / \mathrm{m}^{2}\right)$ & $2.01 \times 10^{-3}$ & - \\
\hline Thermal conductivity, $k(\mathrm{~W} / \mathrm{m} \mathrm{K})$ & 0.613 & 40 \\
\hline Specific heat, $C p(\mathrm{~J} / \mathrm{kg} \mathrm{K})$ & 4182.2 & 765 \\
\hline
\end{tabular}




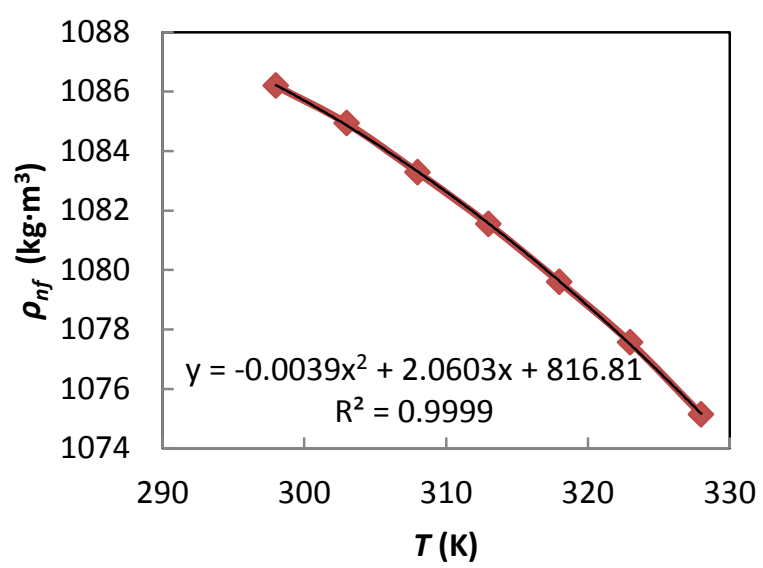

(a)

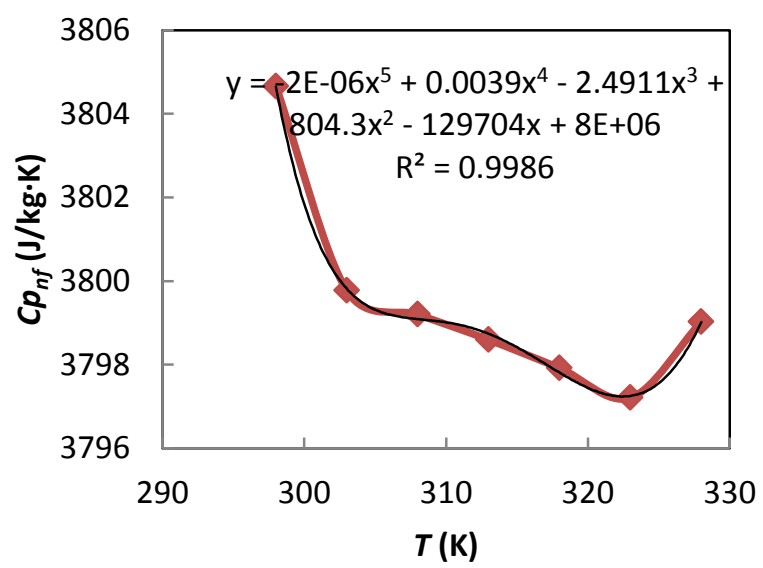

(c)

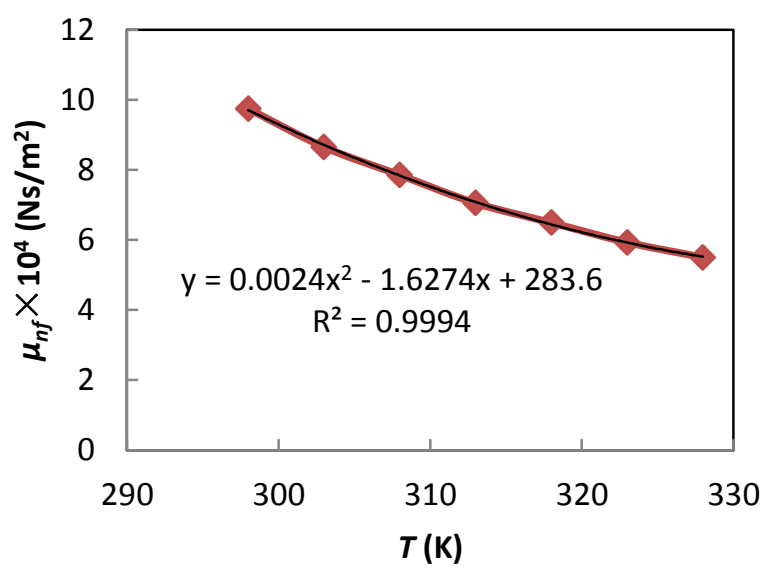

(b)

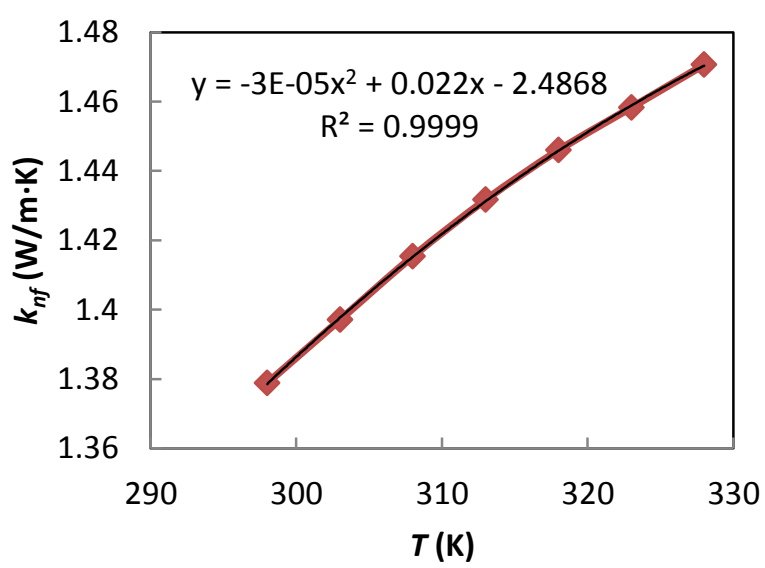

(d)

Fig 2 Curve fitting of nanofluid properties as a function of temperature: (a) Density. (b) Viscosity. (c) Specific heat.

(d) Thermal conductivity.

\section{Governing equations}

The subject is considered to be a 2-D laminar, steady and incompressible flow. This flow is governed by the following continuity equation,

$$
\frac{\partial u}{\partial x}+\frac{\partial v}{\partial y}=0
$$




$$
\begin{gathered}
\rho_{n f}\left(u \frac{\partial u}{\partial x}+v \frac{\partial u}{\partial y}\right)=-\frac{\partial p}{\partial x}+\left[\frac{\partial \tau_{x x}}{\partial x}+\frac{\partial \tau_{y x}}{\partial y}\right] \\
\rho_{n f}\left(u \frac{\partial v}{\partial x}+v \frac{\partial v}{\partial y}\right)=-\frac{\partial p}{\partial y}+\left[\frac{\partial \tau_{x y}}{\partial x}+\frac{\partial \tau_{y y}}{\partial y}\right] \\
\operatorname{div}(T \vec{v})=\operatorname{div}\left(\frac{k_{n f}}{(\rho C p)_{n f}} \operatorname{grad} T\right)
\end{gathered}
$$

For a non-Newtonian power-law fluid, the relationship between the shear stress and shear rate in case of two-dimensional motion in rectangular coordinate are as follows:

$$
\tau=-m\left[\left|\sqrt{\frac{1}{2}(\dot{\gamma} \cdot \dot{\gamma})}\right|^{(n-1)}\right] \dot{\gamma}
$$

where

$$
\frac{1}{2}(\dot{\gamma} \cdot \dot{\gamma})=-2\left[\left(\frac{\partial u}{\partial x}\right)^{2}+\left(\frac{\partial v}{\partial y}\right)^{2}\right]+\left(\frac{\partial u}{\partial y}+\frac{\partial v}{\partial x}\right)^{2}
$$

Thus the stress tensors in Eqs. (2) and (3) change to

$$
\begin{gathered}
\tau_{x x}=-2\left\{m\left|\left\{2\left[\left(\frac{\partial u}{\partial x}\right)^{2}+\left(\frac{\partial v}{\partial y}\right)^{2}\right]+\left(\frac{\partial u}{\partial y}+\frac{\partial v}{\partial x}\right)^{2}\right\}^{1 / 2}\right|^{(n-1)}\right\}\left(\frac{\partial u}{\partial x}\right) \\
\tau_{x y}=\tau_{y x}=-2\left\{m\left|\left\{2\left[\left(\frac{\partial u}{\partial x}\right)^{2}+\left(\frac{\partial v}{\partial y}\right)^{2}\right]+\left(\frac{\partial u}{\partial y}+\frac{\partial v}{\partial x}\right)^{2}\right\}^{1 / 2}\right|^{(n-1)}\right\}\left(\frac{\partial u}{\partial y}+\frac{\partial v}{\partial x}\right) \\
\tau_{y y}=-2\left\{m\left|\left\{2\left[\left(\frac{\partial u}{\partial x}\right)^{2}+\left(\frac{\partial v}{\partial y}\right)^{2}\right]+\left(\frac{\partial u}{\partial y}+\frac{\partial v}{\partial x}\right)^{2}\right\}^{1 / 2}\right|^{(n-1)}\right\}\left(\frac{\partial v}{\partial y}\right)
\end{gathered}
$$

From the former equations, the governing equations for a non-Newtonian power-law fluid will take the following shape:

$$
\frac{\partial u}{\partial x}+\frac{\partial v}{\partial y}=0
$$




$$
\begin{gathered}
\rho_{n f}\left(u \frac{\partial v}{\partial x}+v \frac{\partial v}{\partial y}\right)=-\frac{\partial p}{\partial x}+m\left|\left\{2\left[\left(\frac{\partial u}{\partial x}\right)^{2}+\left(\frac{\partial v}{\partial y}\right)^{2}\right]+\left(\frac{\partial u}{\partial y}+\frac{\partial v}{\partial x}\right)^{2}\right\}^{1 / 2}\right|^{(n-1)}\left(\frac{\partial^{2} u}{\partial x^{2}}+\frac{\partial^{2} u}{\partial y^{2}}\right) \\
\rho_{n f}\left(u \frac{\partial v}{\partial x}+v \frac{\partial v}{\partial y}\right)=-\frac{\partial p}{\partial x}+m\left|\left\{2\left[\left(\frac{\partial u}{\partial x}\right)^{2}+\left(\frac{\partial v}{\partial y}\right)^{2}\right]+\left(\frac{\partial u}{\partial y}+\frac{\partial v}{\partial x}\right)^{2}\right\}^{1 / 2}\right|^{(n-1)} \quad\left(\frac{\partial^{2} v}{\partial x^{2}}+\frac{\partial^{2} v}{\partial y^{2}}\right) \\
\operatorname{div}(T \vec{v})=\operatorname{div}\left(\frac{k_{n f}}{(\rho C p)_{n f}} \operatorname{grad} T\right)
\end{gathered}
$$

The parameters $m$ and $n$ in the former equations are empirical constants, which depends on the type of nanofluid. Putra et al. [40] have shown experimentally the relation between the shear stress and shear strain for $\mathrm{Al}_{2} \mathrm{O}_{3}$-water nanofluid. Using this data, the values of $m$ and $n$ has been calculated for $1 \%$ and $4 \%$ nanoparticle volume fraction. These values are suitably interpolated and extrapolated keeping in mind that the shear stress decreases with increase in $\varphi$ for a particular shear rate in the mixture.

Researchers have found that in a planar 1:3 sudden expansion model, the solution convergence is often a major limitation when the shear-thinning non-Newtonian behavior is enhanced (a smaller $n$ value when $n<1$ ). Poole and Ridley [41] used Fluent software to numerical simulate inelastic power-law fluids and were unable to get a converged solution for $n<0.4$. Ternik [42] used the OpenFOAM software and no converged solutions were obtained for $n<0.6$. Other researchers also reported that the iterative convergence had become increasingly time consuming with the reduction in power-law index. Because of this, the range of the solid fraction $\varphi$ is set to be $0.0 \%$ $\leq \varphi \leq 3.0 \%$ in the present study, so the minimum value of $n$ will not be lower than 0.6 . The values of $m$ and $n$ for different $\varphi$ are given in Table 2.

Table 2 Values of fluid behaviour index parameters $(m, n)$ for $\mathrm{Al}_{2} \mathrm{O}_{3}$-Water nanofluid

\begin{tabular}{|l|l|l|}
\hline$\varphi$ & $m\left(\mathrm{~N} \mathrm{sec}^{\mathrm{n}} \mathrm{m}^{-2}\right)$ & $n$ \\
\hline 0.0 & 0.00100 & 1.000 \\
\hline 1.0 & 0.00230 & 0.830 \\
\hline
\end{tabular}




\begin{tabular}{|l|l|l|}
\hline 2.0 & 0.00347 & 0.730 \\
\hline 3.0 & 0.00535 & 0.625 \\
\hline
\end{tabular}

\section{Boundary conditions}

At the inlet a uniform velocity field is assumed. The nanofluid of temperature $T_{0}$ flows from the left to the right. The downstream of the duct has a constant boundary heat flux rate of $q_{w}$. The outlet boundary condition is set to be pressure outlet. The slip boundary condition is not considered in the present study.

The corresponding boundary conditions can be given as follows:

$$
\begin{aligned}
& u=u_{i n}, v=0, T=T_{0}, \text { at } x=0 \text { and } 0 \leq y \leq h \\
& \frac{\partial u}{\partial x}=v=\frac{\partial T}{\partial x}=0, \text { at } 0 \leq x \leq L_{a} \text { and } 0<y<h \\
& u=v=\frac{\partial T}{\partial x}=0,\left.\frac{\partial T}{\partial y}\right|_{y=0}=q_{w}, \text { at } L_{a} \leq x \leq L_{\text {heat }} \text { and } y=H \\
& u=v=\frac{\partial T}{\partial x}=0, \frac{\partial T}{\partial y}=0, \text { at } L_{\text {heat }} \leq x \leq L_{b} \text { and } y=0
\end{aligned}
$$

\section{Parameter definition}

The former governing equations are solved along with the boundary conditions numerically. Several parameters are calculated from the converged solutions. The $N u_{x}$ and $\overline{N u}$ for the heating wall are defined as follows

$$
\begin{gathered}
N u_{x}=\left.H \frac{k_{n f}}{k_{f}} \frac{\partial T}{\partial y}\right|_{x, 0} \\
N u_{\text {average }}=-\left.\frac{1}{L} \int_{0}^{L} N u_{x} d x\right|_{y=0}
\end{gathered}
$$

The generalized Reynolds number is used in the study to adjust to the non-Newtonian power-law fluid. It is defined based on the upstream channel height and bulk inlet velocity $\left(u_{i n}\right)$ as in previous works with power-law 
fluid flows [42].

$$
\operatorname{Re}_{g e n}=\frac{6 \rho u_{i n}^{(2-n)} h^{n}}{m[(4 n+2) / n]^{n}}
$$

The fully developed pressure drop across the microchannels, $\Delta P$, is defined as:

$$
\begin{gathered}
\Delta P=\Delta P_{\text {in }, \text { out }}-P_{d} \\
P_{d}=1.18 \rho_{n f} u_{\text {in }}
\end{gathered}
$$

\section{Numerical procedure and validation}

\section{Numerical approach}

The computational fluid dynamics code Fluent in the commercial software package ANSYS 15.0 is used in this study. The system of governing equations (17) - (20) are solved by control volume approach. The extra-stress tensor brought by the non-Newtonian effect are added using the UDF function in the software. Second order upwind scheme is employed to discretize the convection terms, diffusion terms and other quantities resulting from the governing equations. The convective terms are discretized with the QUICK scheme. The pressure and velocity were coupled using the standard PISO algorithm. For all the simulations performed in the study, the convergence criteria are set to be $10^{-5}$ for the residuals of the continuity equation and the momentum equation and $10^{-9}$ for that of the energy equation.

\section{Grid independency and Validation}

The computational domain is mapped by structured meshes and is partitioned into five blocks as shown in Fig.

3. In the streamwise direction the grids are stretched in geometric progression in each block, whereas in the transverse direction they are uniform. The grids are finer near the step of the expansion and are coarser near the inlet and outlet. Four grids were used in order to check the grid dependence on the results. They were named 
meshes A, B, C and D as detailed in Table 3.The four meshes were differentiated by fineness. Mesh A is the coarsest while Mesh D is the finest.

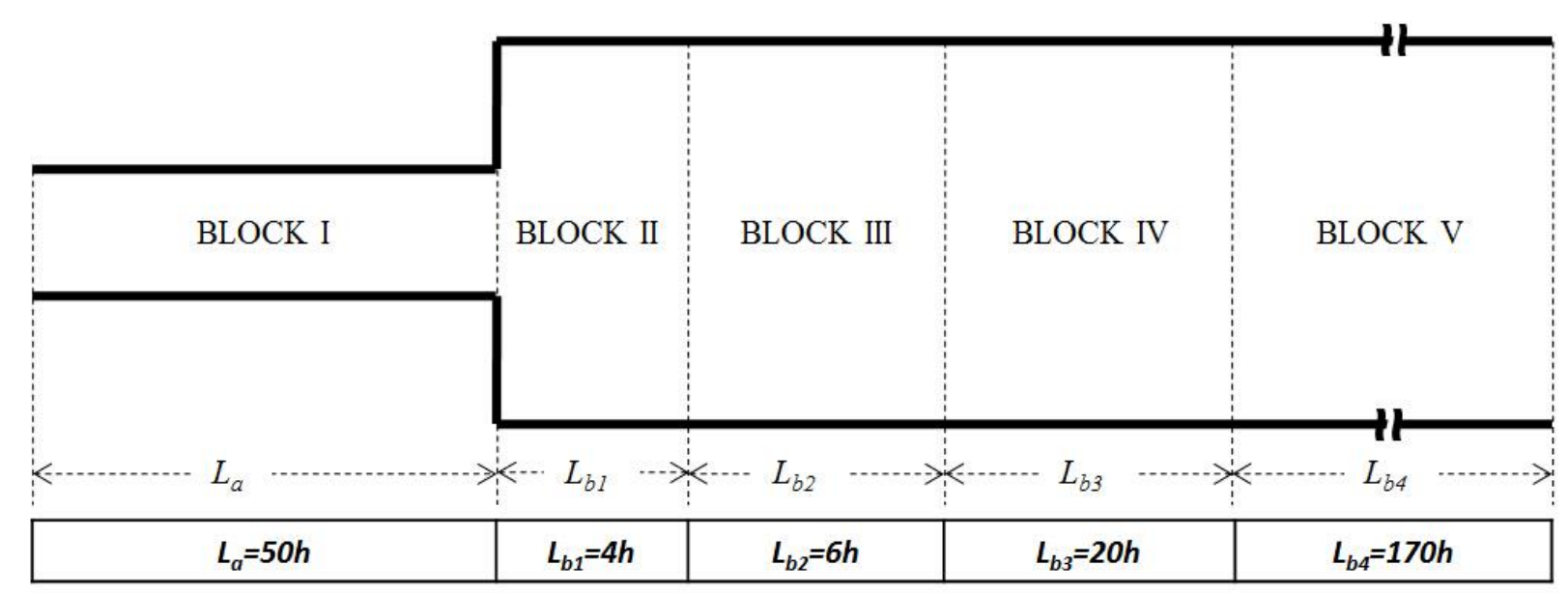

Fig 3 Illustration of blocks that were used.

Table 3 Computational domain and mesh characteristics of the geometry

\begin{tabular}{|l|l|l|l|l|l|}
\hline Mesh \# & Mesh $A$ & Mesh $B$ & Mesh $C$ & Mesh D \\
\hline$\Delta x_{\min } / h$ & 0.08 & 0.04 & 0.02 & 0.01 \\
\hline$\Delta y_{\min } / h$ & 0.08 & 0.04 & 0.02 & 0.01 \\
\hline \multirow{3}{*}{$N_{x} \times N_{y}$} & BLOCK I & $72 \times 13$ & $144 \times 25$ & $288 \times 51$ & $576 \times 102$ \\
\cline { 2 - 6 } & BLOCK II & $35 \times 39$ & $70 \times 75$ & $140 \times 153$ & $280 \times 306$ \\
\cline { 2 - 6 } & BLOCK III & $38 \times 39$ & $76 \times 75$ & $152 \times 153$ & $304 \times 306$ \\
\cline { 2 - 6 } & BLOCK IV & $63 \times 39$ & $126 \times 75$ & $252 \times 153$ & $504 \times 306$ \\
\cline { 2 - 6 } & BLOCK V & $75 \times 39$ & $150 \times 75$ & $300 \times 153$ & $600 \times 306$ \\
\hline \multirow{5}{*}{ Total Size } & & $\mathbf{9 , 1 6 5}$ & $\mathbf{3 5 , 2 5 0}$ & $\mathbf{1 4 2 , 8 8 0}$ & $\mathbf{5 7 5 , 2 8 0}$ \\
\hline
\end{tabular}

Because the flow pattern of non-Newtonian fluid is much more sensitive to the change of the mesh size than the heat transfer performance, we used the vortex characteristics obtained at various generalized Reynolds numbers (a parameter that reflects the exact flow pattern) to check the grid dependence.

Table 4 presents the vortex characteristics at $\varphi=0.03(n=0.650)$ obtained using different meshes for three 
different generalized Reynolds numbers in the study. Each of the generalized Reynolds numbers represents a different flow regime, i.e. the symmetric flow, the asymmetric flow and the asymmetric flow with a third eddy. The results shows that nearly grid independent results, to within $3 \%$ of the refined Mesh $\mathrm{D}$, could be obtained using Mesh C. As such, the subsequent results in this study were obtained using mesh C.

Furthermore, Table 5 shows the comparison between the vortex characteristics obtained using Mesh $\mathrm{C}$ and those obtained by S. Dhinakaran [43] at $\varphi=0.00(n=1.000)$. The results obtained in the current study are in good agreement with those predicted by S. Dhinakaran with a maximum percentage error of $0.2 \%$.

Table 4 Mesh dependence check

\begin{tabular}{|c|c|c|c|c|c|c|c|c|}
\hline \multirow{2}{*}{$\varphi=0.03$} & \multicolumn{2}{|c|}{$R e_{g e n}=80$} & \multicolumn{2}{|c|}{$R e_{\text {gen }}=180$} & \multicolumn{4}{|c|}{$R e_{g e n}=360$} \\
\hline & $X_{a} / h$ & $X_{b} / h$ & $X_{a} / h$ & $X_{b} / h$ & $X_{a} / h$ & $X_{b} / h$ & $X_{c} / h$ & $X_{d} / h$ \\
\hline Mesh A & 3.5097 & 3.5337 & 7.4981 & 7.8576 & 17.9801 & 4.6801 & 14.35 & 28.46 \\
\hline Mesh B & 3.2312 & 3.2462 & 6.7675 & 6.7751 & 15.9421 & 4.5899 & 15.01 & 19.14 \\
\hline Mesh C & 3.1514 & 3.1514 & 6.7638 & 6.7507 & 16.9261 & 4.8311 & 15.86 & 19.76 \\
\hline Mesh D & 3.1113 & 3.1038 & 6.7579 & 6.7505 & 16.9389 & 4.9702 & 15.88 & 19.81 \\
\hline
\end{tabular}

Table 5 Method validation: comparison of the predicted vortex size in this work with those obtained by S.

Dhinakaran [43] at $\operatorname{Re}_{\text {gen }}$ from 50-120, $\varphi=0.00$.

\begin{tabular}{|l|l|l|l|l|l|l|l|l|}
\hline \multirow{2}{*}{$\varphi=0.00$} & \multicolumn{3}{|l|}{$\operatorname{Re}_{\text {gen }}=50$} & \multicolumn{2}{l|}{$\operatorname{Re}_{\text {gen }}=70$} & $R_{\text {gen }}=120$ & \\
\cline { 2 - 9 } & $X_{a} / h$ & $X_{b} / h$ & $X_{d} / h$ & $X_{b} / h$ & $X_{d} / h$ & $X_{b} / h$ & $X_{d} / h$ & $X_{d} / h$ \\
\hline S. Dhinakaran, 2013 & 5.0873 & 5.0873 & 9.0495 & 3.6742 & 12.8721 & 3.9643 & 11.2647 & 16.9065 \\
\hline Present Study $($ Mesh C) & 5.0910 & 5.091 & 9.0661 & 3.6772 & 12.7937 & 3.9624 & 11.147 & 16.9917 \\
\hline
\end{tabular}

\section{Results and discussion}

Flow pattern of nanofluid at different nanoparticle volume fractions

The variation of recirculation length at the downstream of the expansion with the generalized Reynolds number for the entire range investigated is presented in Fig. 4a-d for different values of the nanoparticle volume 
fraction of nanofluid. Initially, at low generalized Reynolds number, the recirculating eddies are symmetric and grow with an increase in the generalized Reynolds number (their lengths along the walls are referred to as $X_{a}$ and $X_{b}$ as shown in Fig. 1b). When the generalized Reynolds number reaches a critical value $\left(R e_{g e n, c r l}\right)$, one vortex grows larger than the other. There is no preferred wall for this to happen, but for the sake of simplification we consider that $X_{a}>X_{b}$. With a further increase in the generalized Reynolds number, a third eddy appears on the side of the smaller one of the two main vortexes and is located further downstream. The critical generalized Reynolds number at which this third eddy appears is defined as the second critical Reynolds number $\left(\operatorname{Re}_{g e n, c r 2}\right)$. The distance from the step to the point where the flow separates to form the third eddy is termed $X_{c}$ and the distance from the step to the point where the flow reattaches on the wall is termed $X_{d}$ as sketched in Fig. $1 \mathrm{~b}$.

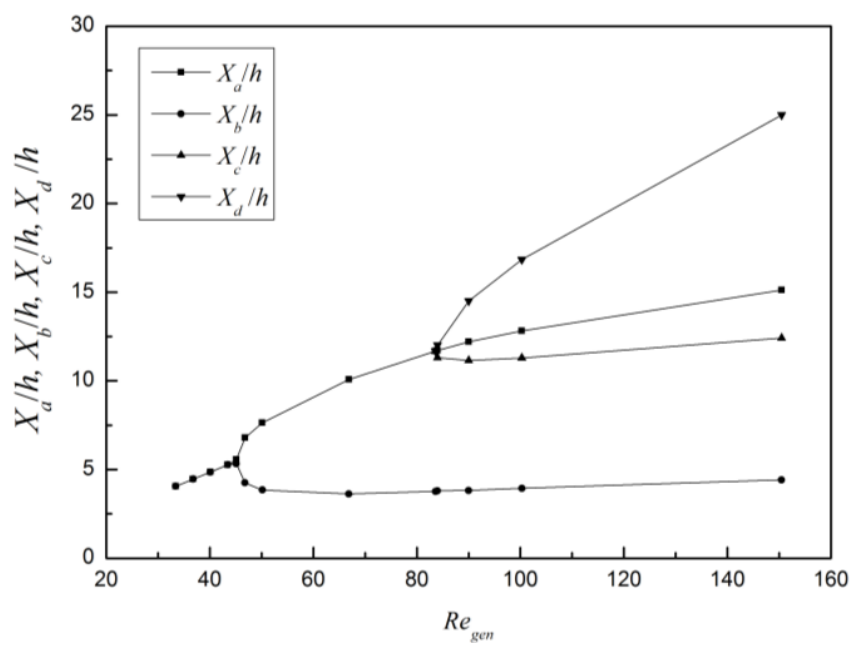

(a)

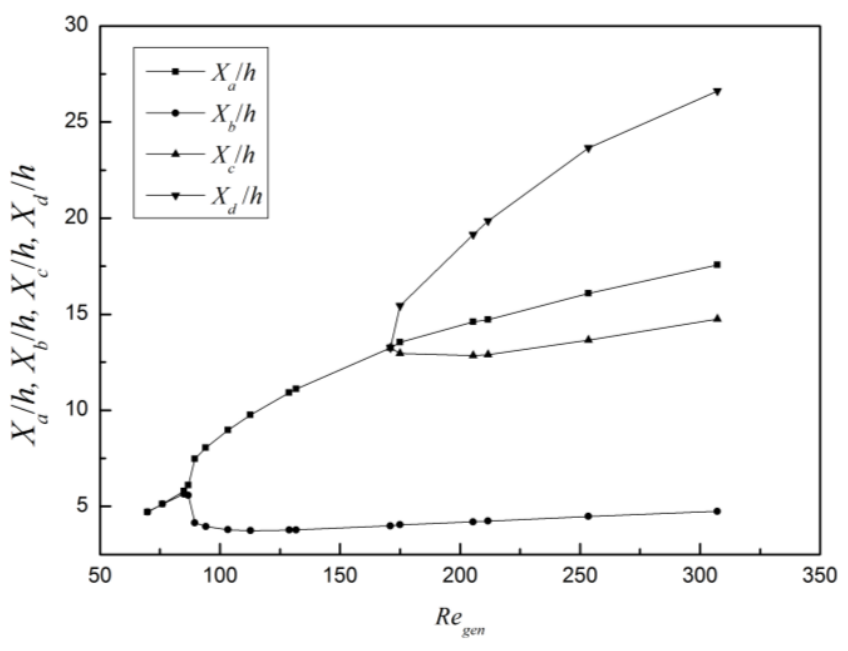

(b) 


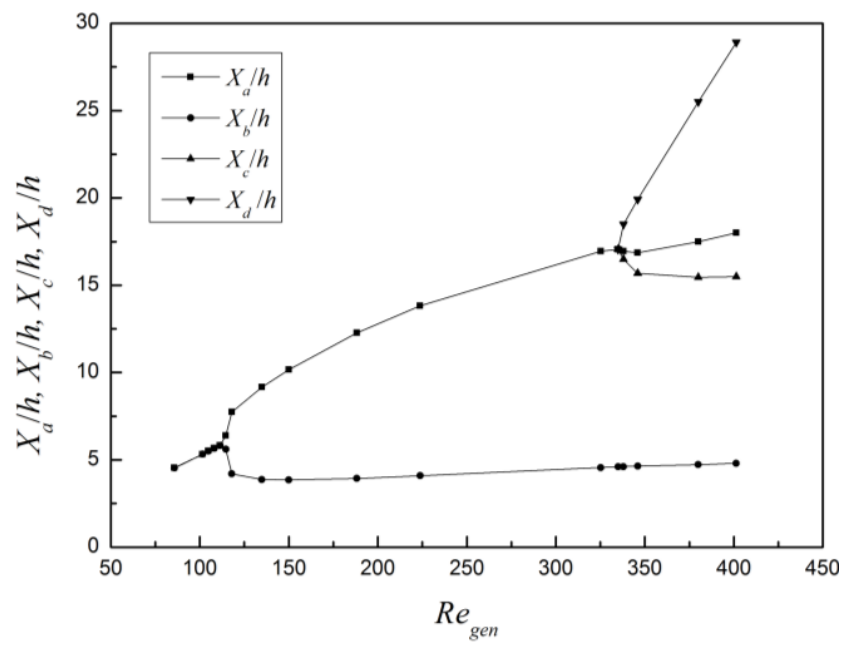

(c)

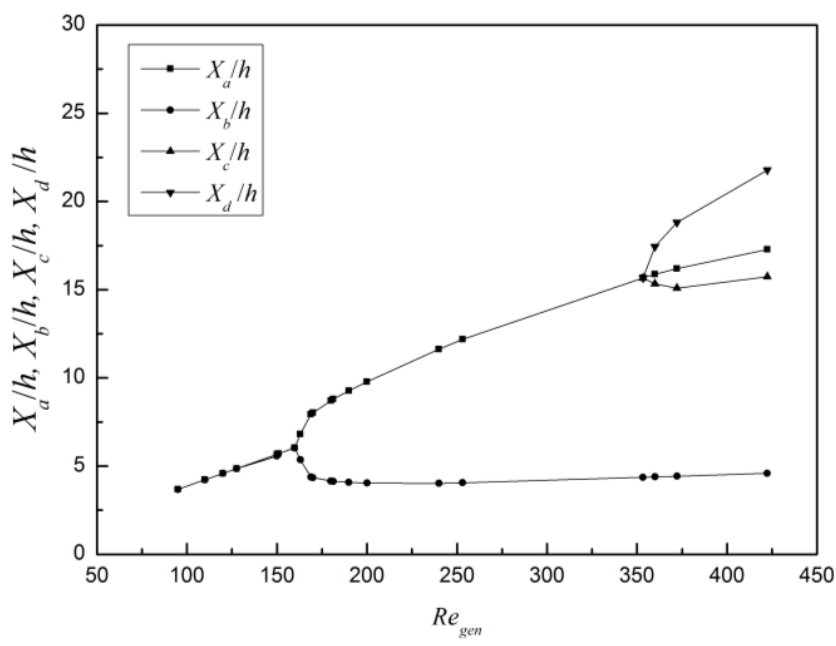

(d)

Fig 4 Variation of vortex size with generalized Reynolds number for the power-law nanofluid flow in a 1:3 planar sudden expansion with the wall heat transfer rate of $q_{w}=150 \mathrm{~kW} / \mathrm{m}^{2}$ at different nanoparticle volume fraction values: (a) $\varphi=0 \%$; (b) $\varphi=1 \%$; (c) $\varphi=2 \%$; (d) $\varphi=3 \%$

For $\varphi=0 \%(n=1)$, which can be treated as Newtonian fluid, the first critical Reynolds number is $R e_{g e n, c r l}=46$. Above this value of $R e_{g e n}$, the flow becomes asymmetric. The longer eddy $\left(X_{a}\right)$ continues to grow in size and smaller eddy $\left(X_{b}\right)$ continues to decrease, until $R e_{g e n, c r 2}=88$ when the third eddy appears. The two critical numbers obtained in the present work are about $20 \%$ smaller than the results of Ternik [42]. This is because the heating at the side wall accelerate the formation of the vortexes. The viscosity of the fluid decreases as the temperature increases, which leads to an earlier bifurcation of the fluid. Comparing the results of $X_{a}, X_{b}, X_{c}$ and $X_{d}$ for $\varphi=0 \%(n$ $=1)$ and $\varphi=1 \%(n=0.830)$, it can be seen that shearing-thinning delays all the flow transitions. For $\varphi=1 \%$ under the current boundary conditions, the onset of asymmetry is delayed to $R e_{g e n, c r l}=86.8$, and the appearance of the third eddy is delayed to $R e_{g e n, c r 2}=177$. Similarly, for $\varphi=2 \%$, the onset of asymmetry is delayed to $\operatorname{Re} e_{\text {gen, } c r l}=$ 111.4, and the appearance of the third eddy is delayed to $R e_{g e n, c r 2}=335$. Further increasing of the solid particle 
fraction of the nanofluid, corresponding to stronger shearing-thinning, and results in further delayed for transitions

$\left(R e_{g e n, c r l}=160\right.$ and $R e_{g e n, c r 2}=353$ for $\left.\varphi=3 \%\right)$.

(a)

(i)

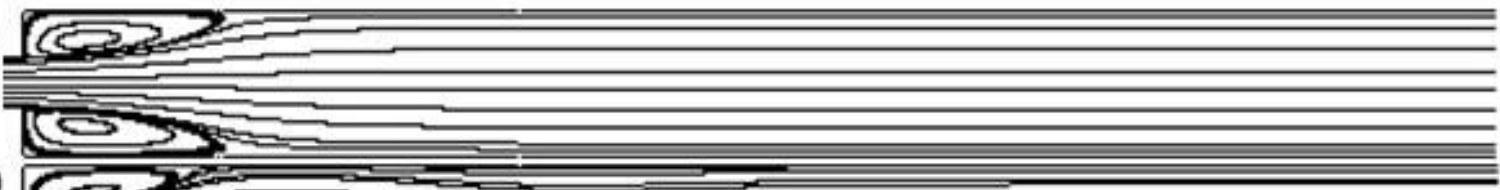

(ii)

20

$T+2$

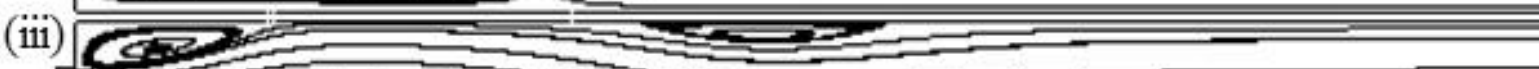
$\Rightarrow=2=$ $T<2=0.2$

(i)

$\rightarrow 3$

(ii) $\mathrm{arr}_{2} \mathrm{x}$

(b)

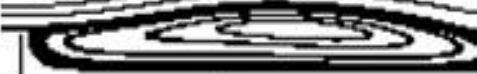

(iii) $\Rightarrow$ F $\Rightarrow=0$

(i) $\Rightarrow=0$

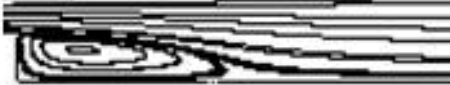

(c)

(ii)

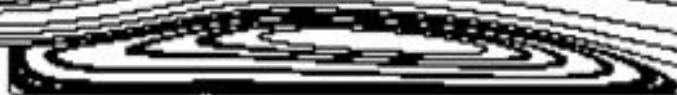

(iii)

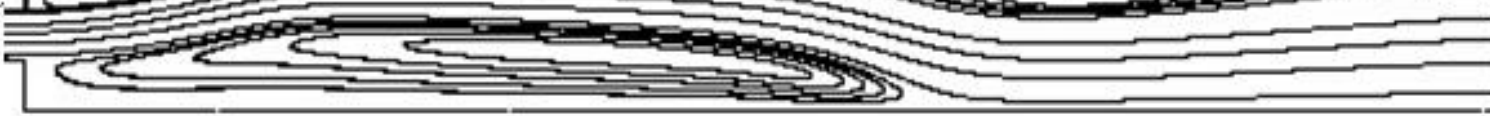

(i)

(10을

(ii)

$3=2$

(d)

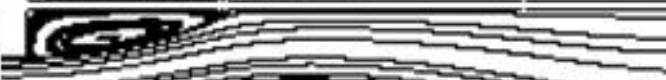

$\mathrm{T}=\mathrm{z}=\mathrm{m}$

(iii)

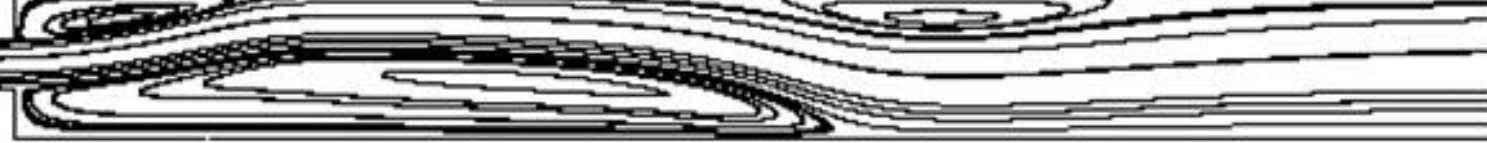

Fig 5 Flow patterns in the 1:3 planar sudden expansion at three different regimes for each case: symmetric flow 
$\left(R e_{\text {gen }}=0.8 R e_{\text {gen, } c r l}\right)$; asymmetric flow $\left[R e_{g e n}=0.5\left(R e_{g e n, c r l}+R e_{g e n, c r 2}\right)\right]$; asymmetric flow with a third recirculating eddy $\left(R e_{g e n}=1.2 R e_{g e n, c r 2}\right)$ at different nanoparticle volume fraction values: (a) $\varphi=0 \%$; (b) $\varphi=1 \%$; (c) $\varphi=2 \% ;(\mathrm{d}) \varphi=3 \%$.

To illustrate the flow patterns in different regimes, the streamline plots are depicted in Fig. 5a-d for different nanoparticle volume fraction values. Since there is a strong variation of $\operatorname{Re}_{g e n, c r 1}$ and $\operatorname{Re} e_{g e n, c r 2}$ at different $\varphi$, the values of generalized Reynolds numbers used are qualitatively the same in the following sense: for the symmetric regime we considered a value of $R e_{g e n}=0.8 R e_{g e n, c r l}$, for the asymmetric regime we considered a value of $R e_{g e n}=$ $0.5\left(R e_{g e n, c r 1}+R e_{g e n, c r 2}\right)$, for the asymmetric with a third eddy regime we considered a value of $R e_{g e n}=1.2 R e_{g e n, c r 2}$. It can be seen that the vortexes sizes are gradually become larger as $\varphi$ increases, with a slightly different case of $\varphi=$ $2 \%$.

For nanofluid, the shear-thinning effect becomes stronger, while the fluid viscosity becomes larger as the nanoparticle volume fraction $\varphi$ increases. From the delay of the critical generalized Reynolds number we can see that the shear-thinning phenomenon stabilizes the flow when comparing to the Newtonian flow case. In the planar sudden expansion, a perturbation to the symmetric flow at the step pushes the fluid towards one of the sides of the expansion. This gives a rise to the local velocities and leads to a lower local pressure, and gradually form the asymmetric eddies. For a smaller $n$, the raise of the near wall velocity brought from the perturbation become smaller, which leads to a later critical $R e_{\text {gen }}$ and a larger eddy size. While for a larger viscosity, the size of the eddy formed near the wall will first increase then decrease before they are washed off. This two phenomena add together, leading to an abnormal increase of the vortexes size at $\varphi=2 \%$.

The pressure drop changes with $R e_{\text {gen }}$ at different nanoparticle volume fraction values are plotted in Fig. 6 . We can see a significant decrease of pressure drop as the nanoparticle volume fraction increases. For the Newtonian 
fluid case $(\varphi=0 \%)$, the pressure drop increases linearly with the generalized Reynolds number. As the nanoparticle volume fraction values increase, the relationship between the pressure drop and the generalized Reynolds number become much more nonlinear.

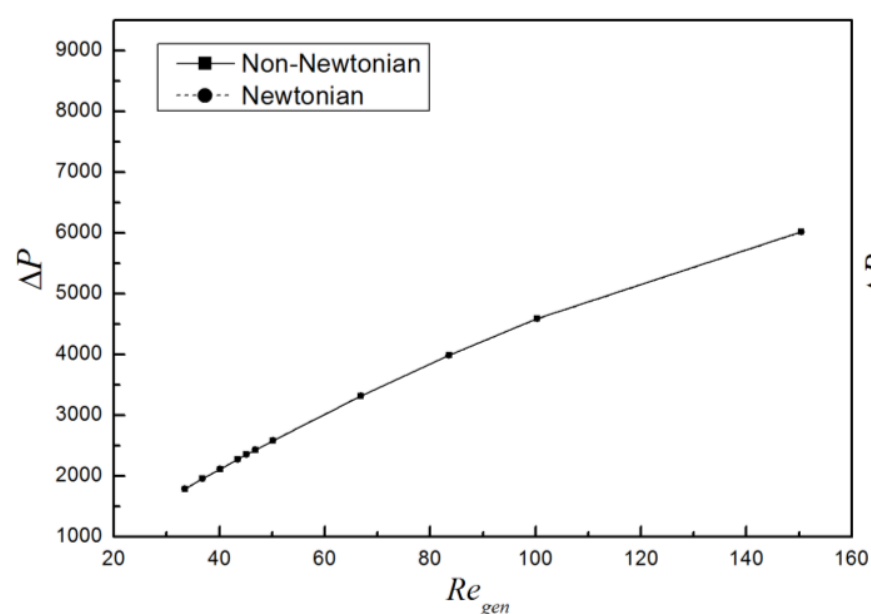

(a)

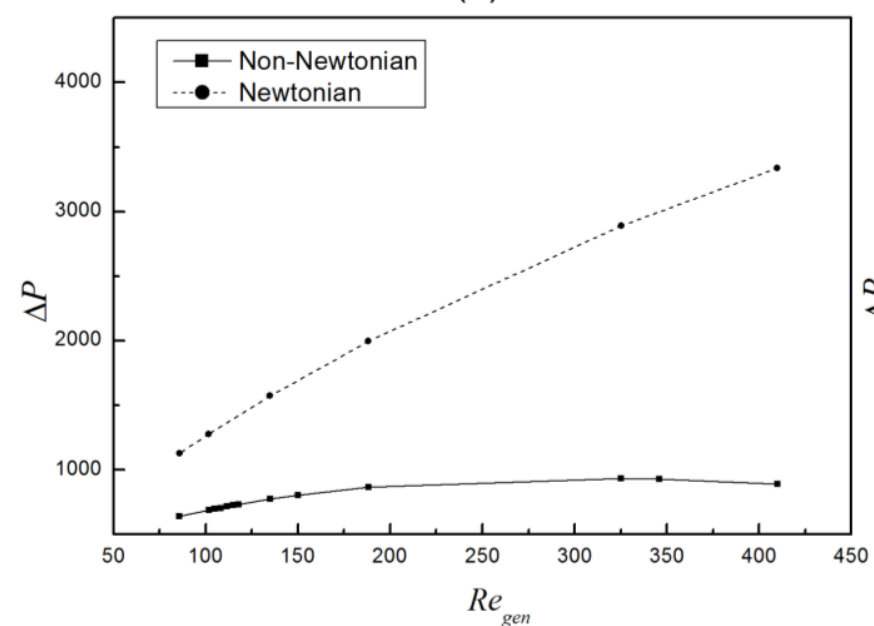

(c)

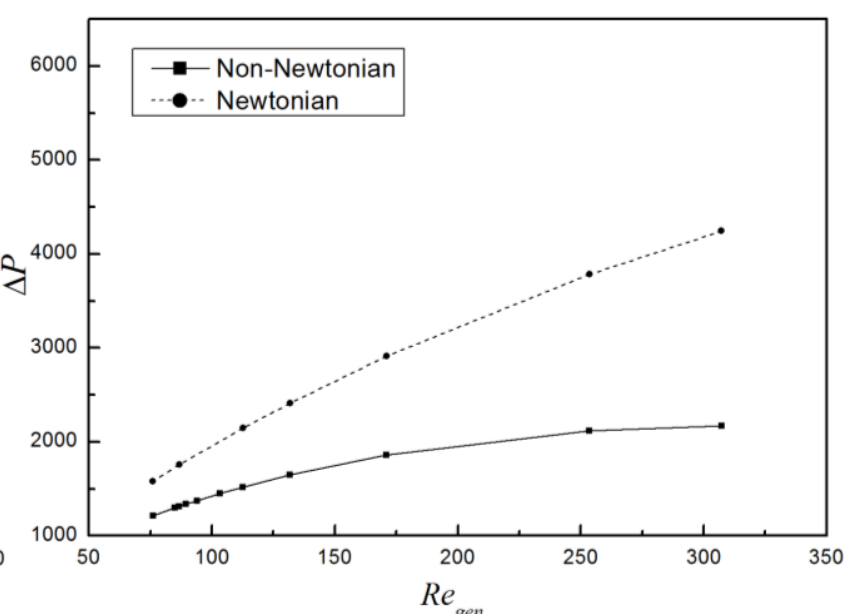

(b)

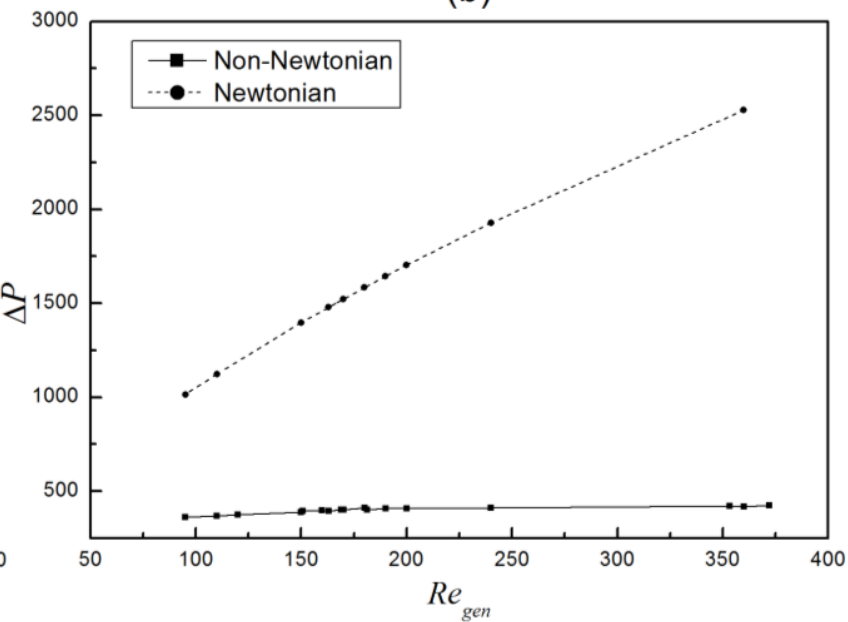

(d)

Fig 6 Pressure drop with $R e_{g e n}$ at different nanoparticle volume fraction values: (a) $\varphi=0 \%$; (b) $\varphi=1 \%$; (c) $\varphi=2 \%$;

(d) $\varphi=3 \%$ using Non-Newtonian model/ Newtonian model.

In order to evaluate the differences brought from using the non-Newtonian model, we also investigate the pressure drop (defined in Eq. (24)) of difference inlet generalized Reynolds number obtained using Newtonian model. In the Newtonian cases, the fluid remains the same thermophysical properties but the flow fields are solved 
using Newtonian equations. We can see that for $\varphi=0 \%$, which is actually a Newtonian fluid, the two lines appear as one. As the nanoparticle volume fraction $\varphi$ increases (which means the shear-thinning effect increases), the gap between the non-Newtonian curves and the Newtonian curves becomes widen. Especially, there will be about 50\% over estimate of pressure drop at $\varphi=1 \%$ in the considered $R e_{\text {gen }}$ range, which is in good agreement with the work done by Kherbeetet. al [10]. It is clear that using Newtonian model to solve the nanofluid problems will lead to a significant over estimate of pressure drop.

\section{Heat transfer performance of nanofluid at different nanoparticle volume fractions}

The heat transfer performance of nanofluid at different nanoparticle volume fractions at three different flow pattern regimes are illustrated in Fig. 7. The values of the generalized Reynolds numbers used are qualitatively the same as stated before. Because of the shear-thinning effect, the nanofluid of a higher nanoparticle volume fraction can reach to the same flow pattern at a much lower inlet velocity, which leads to a much higher overall temperature of the flow field. Beside of that, the temperature distributions are strongly related to the velocity field. There exists a significant local temperature increase in the recirculation zone. 
(a)

(i)

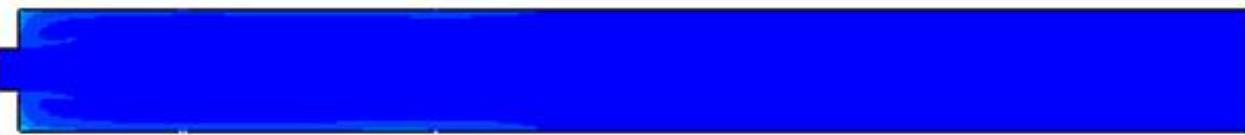

(ii)

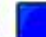

(iii)

(iii)

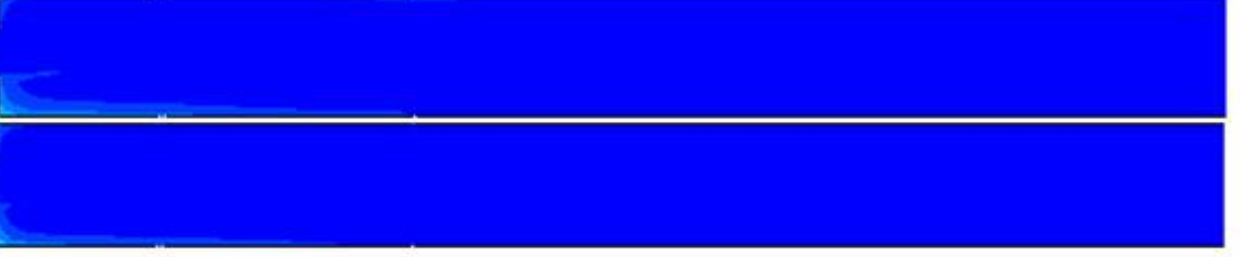

(i)

(b)

(ii)
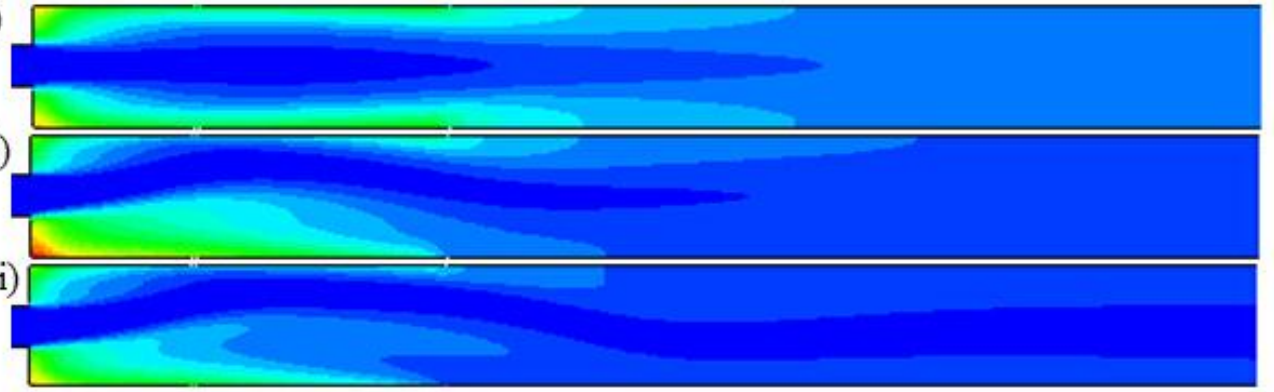

(c)

(i)

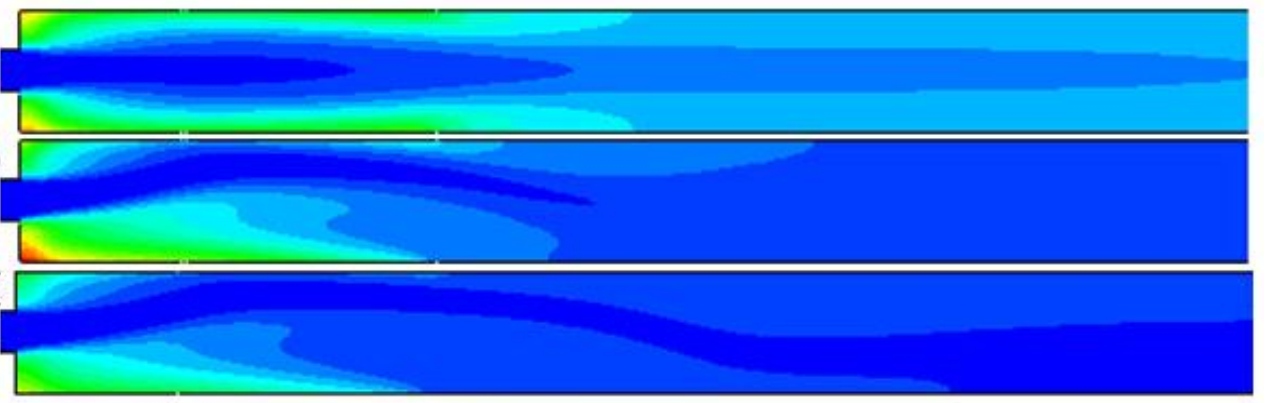

(i)

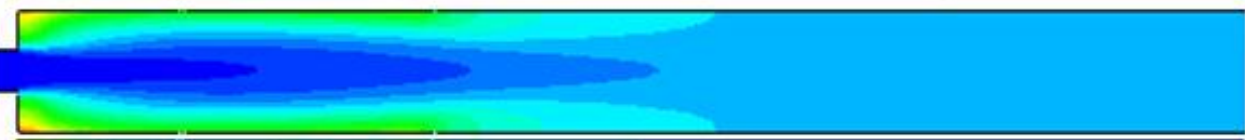

(ii)

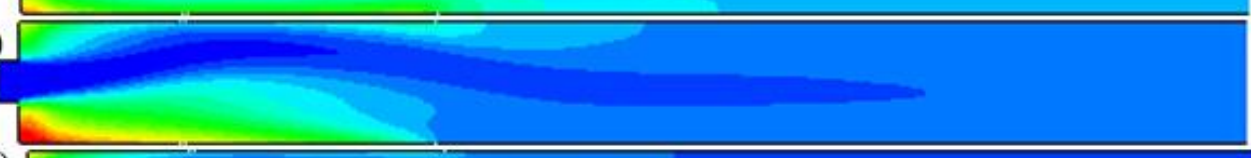

(iii)

(d)

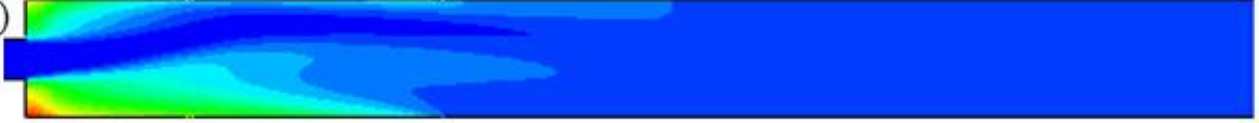

\begin{tabular}{|c|c|}
\hline Temperature \\
$317(\mathrm{~K})$ \\
316 \\
315 \\
3314 \\
313 \\
312 \\
312 \\
311 \\
310 \\
309 \\
308 \\
\hline 307 \\
306 \\
305 \\
304 \\
303 \\
302 \\
301 \\
\hline
\end{tabular}

Fig 7 Temperature distributions in the 1:3 planar sudden expansion at three different regimes for each case:

symmetric flow $\left(R e_{g e n}=0.8 R e_{g e n, c r l}\right)$; asymmetric flow $\left[R e_{g e n}=0.5\left(R e_{g e n, c r l}+R e_{g e n, c r 2}\right)\right]$; asymmetric flow with a third recirculating eddy $\left(\operatorname{Re}_{\text {gen }}=1.2 R e_{\text {gen, } r r 2}\right)$ at different nanoparticle volume fraction values: (a) $\varphi=0 \%$; (b) $\varphi=$ $1 \%$; (c) $\varphi=2 \%$; (d) $\varphi=3 \%$

In order to learn about the details of the heat transfer performance of the three different regimes at each nanoparticle volume fraction value, we investigate the local Nusselt number (plotted in Fig. 8) and average Nusselt 
number (listed in Table 6) of each case. The x-coordinate in Fig. 8 is the relative position of the expansion step. As discussed before, the increase of the inlet $R e_{g e n}$ can result in the change of the flow pattern. Generally, one side of the vortexes becomes larger, stretching to the downstream, and then the third vortex appears in the distance. The generation of the vortexes can enhance the local heat transfer performance in some sense (the peak value of $N u_{x}$ in each figure), but there will also be a deterioration area right after. The primary vortex rotates clockwise while the secondary and the tertiary vortexes rotate anticlockwise, thus the flow direction of the fluid near the mainstream is parallel to the mainstream. The flow direction of the vortexes makes it difficult to transfer heat outside the zone and in this way, weakens the local heat transfer performance. For the Newtonian fluid case $(\varphi=0 \%)$, we can see a notable decrease of the local Nusselt number at the recirculation zone. As the inlet $R e_{\text {gen }}$ increases, the primary vortex grows and makes the local heat transfer performance much worse. Also, the appearance of the tertiary vortex reduces the local Nusselt number of the second half. But as the nanoparticle volume fraction value increases, the gaps among the three regimes narrow. The reduction of the local Nusselt number brought from the primary vortex and the tertiary vortex is not that significant, and another peak appears at the end of the primary vortex. Basically, this is because that the thermophysical properties of the nanofluid change the local heat transfer mechanism. The average Nusselt number of each case in Table 6 also show that the heat transfer deterioration brought by flow pattern change becomes inconspicuous as the solid particle volume fraction $\varphi$ increases.

Table 6 The average Nusselt number of each flow regime at different nanoparticle volume fractions

\begin{tabular}{|c|c|c|c|c|c|}
\hline \multirow{2}{*}{$\varphi$} & symmetric flow & \multicolumn{2}{|c|}{ asymmetric flow } & \multicolumn{2}{|c|}{ asymmetric flow with a third eddy } \\
\cline { 2 - 6 } & $N u_{\text {average }}$ & $N u_{\text {average }}$ & $\begin{array}{c}\text { Decrease of } N u_{\text {average }} \text { comparing } \\
\text { to symmetric flow }\end{array}$ & $N u_{\text {average }}$ & $\begin{array}{c}\text { Decrease of } N u_{\text {average }} \text { comparing } \\
\text { to symmetric flow }\end{array}$ \\
\hline $0 \%$ & 97.50 & 96.94 & $-0.58 \%$ & 96.54 & $-0.99 \%$ \\
\hline $1 \%$ & 73.26 & 73.07 & $-0.20 \%$ & 73.02 & $-0.25 \%$ \\
\hline $2 \%$ & 73.29 & 73.15 & $-0.14 \%$ & 73.12 & $-0.17 \%$ \\
\hline $3 \%$ & 73.32 & 73.26 & $-0.06 \%$ & 73.19 & $-0.13 \%$ \\
\hline
\end{tabular}




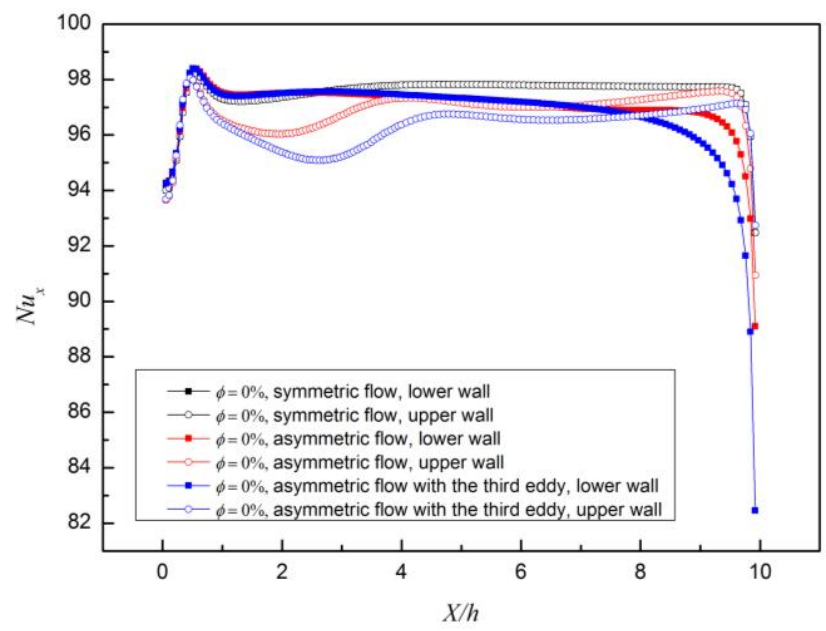

(a)

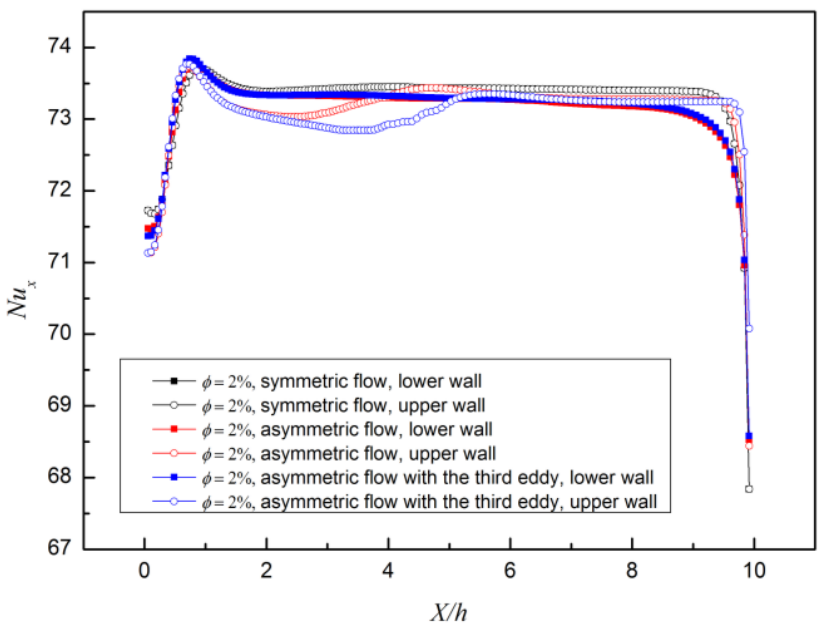

(c)

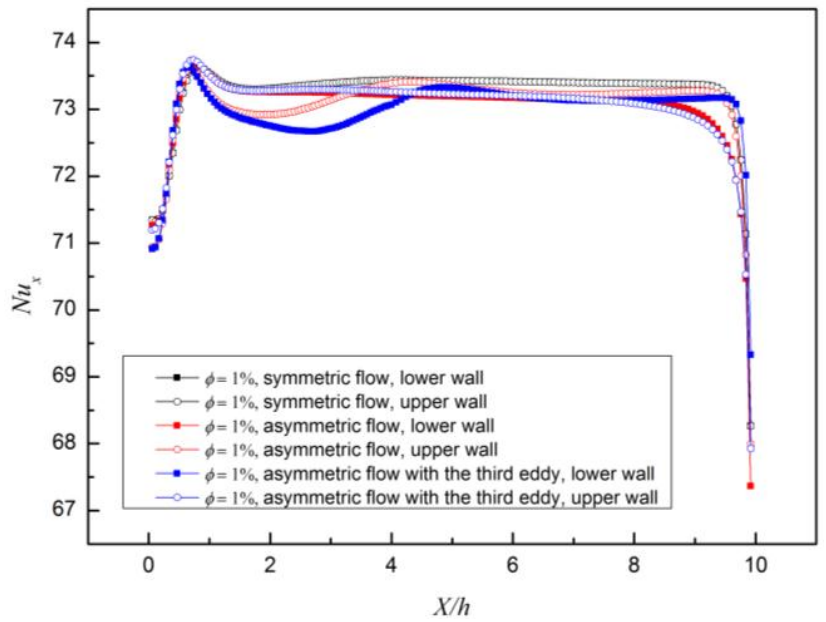

(b)

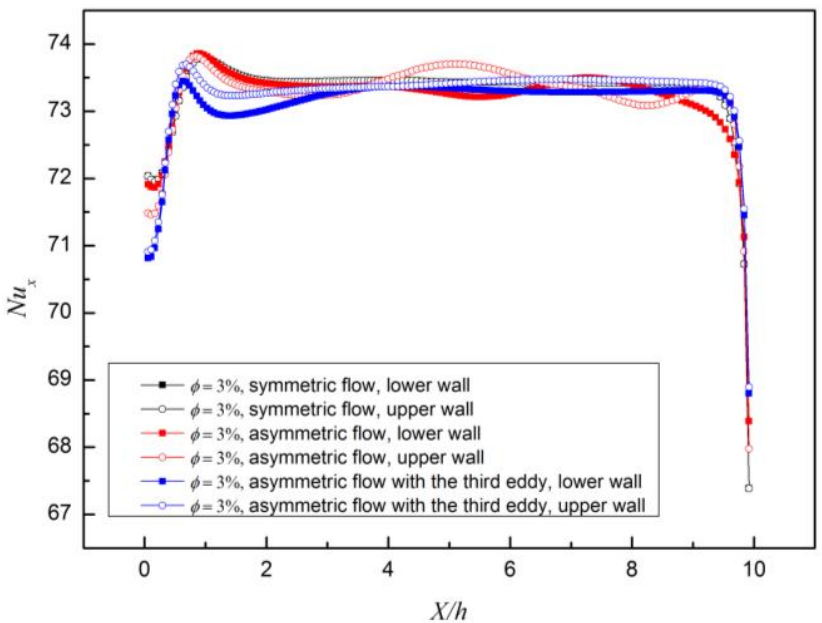

(d)

Fig 8 Local Nusselt number with $x$-axis location at different nanoparticle volume fraction values: (a) $\varphi=0 \%$; (b) $\varphi$

$=1 \% ;$ (c) $\varphi=2 \% ;$ (d) $\varphi=3 \%$

The influence of $q_{w}$ on the flow and heat transfer of nanofluid

The influence brought by the wall heat transfer rate $q_{w}$ on the flow and heat transfer of nanofluid is also studied in the present work. Fig. 9 depicts the flow patterns in the geometry at three different regimes for each case: symmetric flow; asymmetric flow; asymmetric flow with a third recirculating eddy at $\varphi=3 \%$ at a heat transfer rate values range from $100 \mathrm{~kW} / \mathrm{m}^{2}$ to $200 \mathrm{~kW} / \mathrm{m}^{2}$. The nanofluid considered in the current study is temperature 
dependent. The viscosity of the nanofluid decreases as temperature increases. So generally in Fig. 9, the vortexes generated under a higher heat flux rate are shorter and more curve than those generated under a lower heat flux rate and this phenomena is more significant under higher $R e_{g e n}$. The temperature fields for each case are illustrated in

Fig. 10. The temperature fields are strongly related to the flow fields.

(a)

(i)

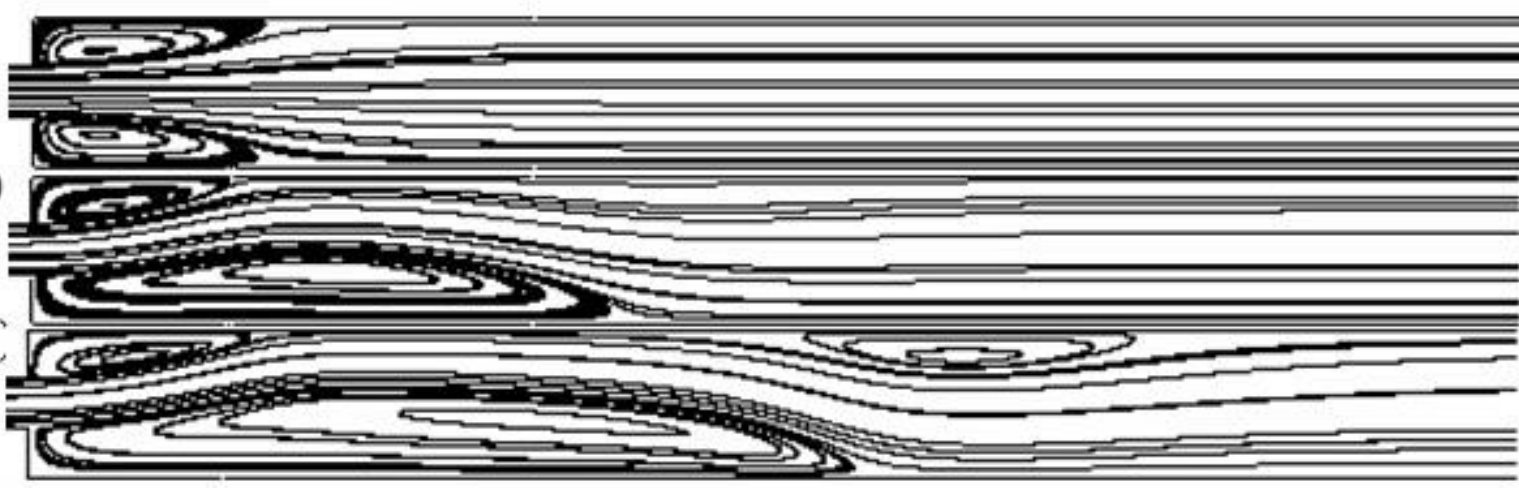

(i)

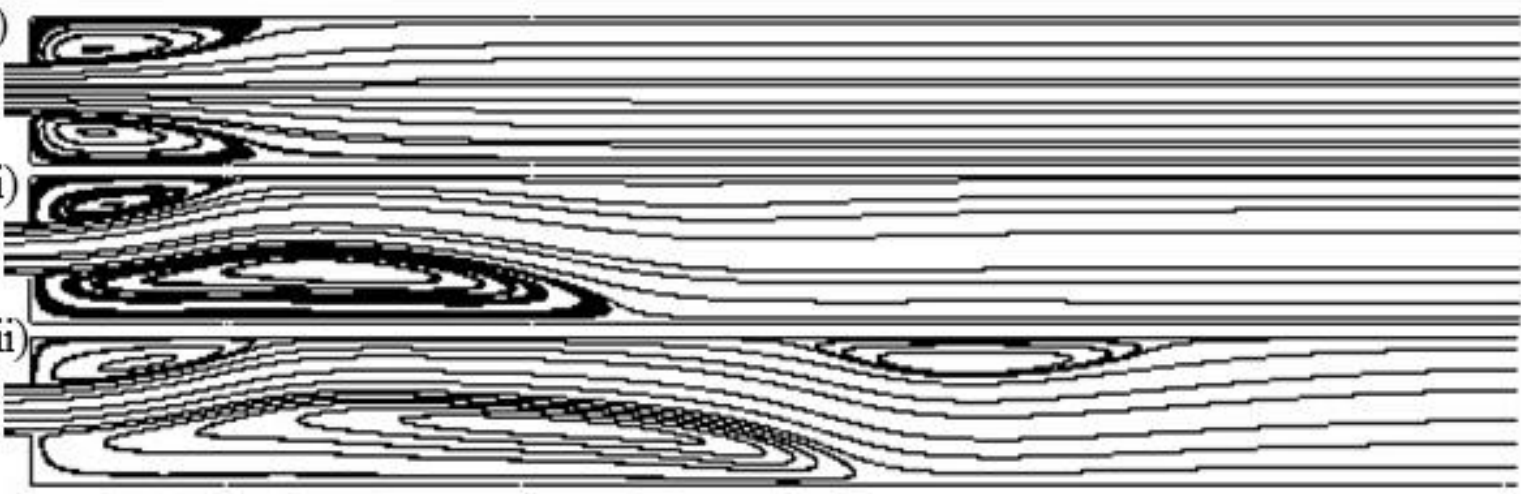

(b)

(ii)

(i)

(c)

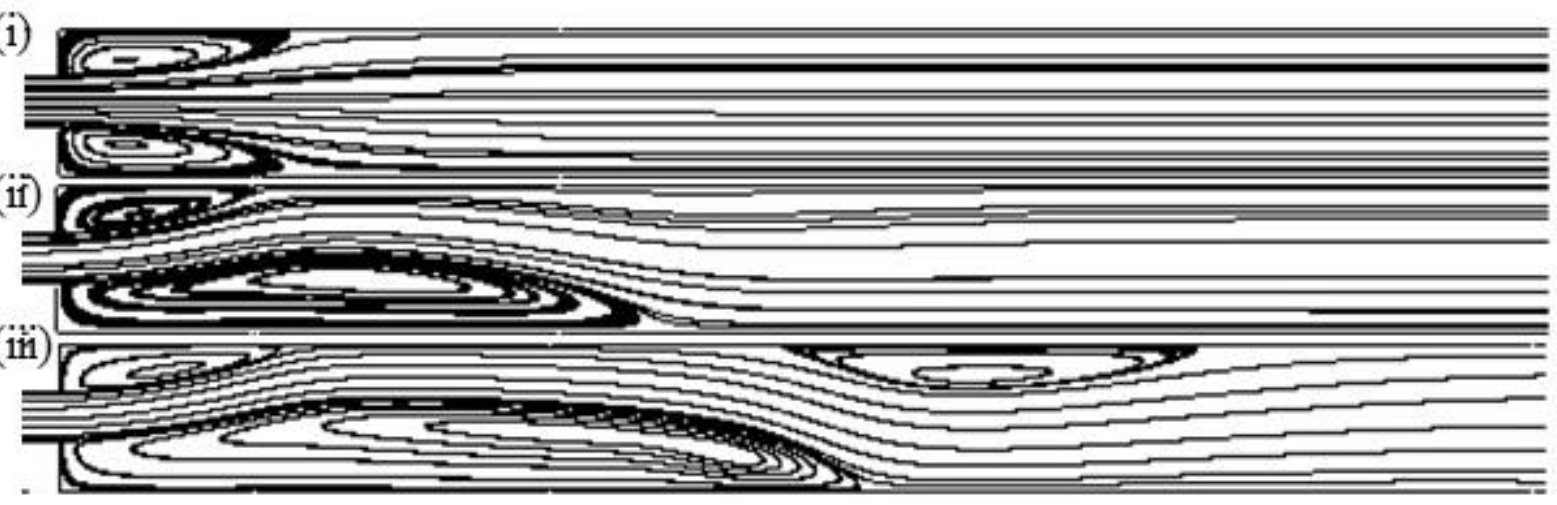

Fig 9 Flow patterns in the 1:3 planar sudden expansion at three different regimes for each case: symmetric flow;

asymmetric flow; asymmetric flow with a third recirculating eddy at different heat transfer rate values: (a) $q_{w}=$

$100 \mathrm{kw} / \mathrm{m}^{2} ;$ (b) $q_{w}=150 \mathrm{kw} / \mathrm{m}^{2} ;$ (c) $q_{w}=200 \mathrm{kw} / \mathrm{m}^{2} .(\varphi=3 \%)$ 
(a)

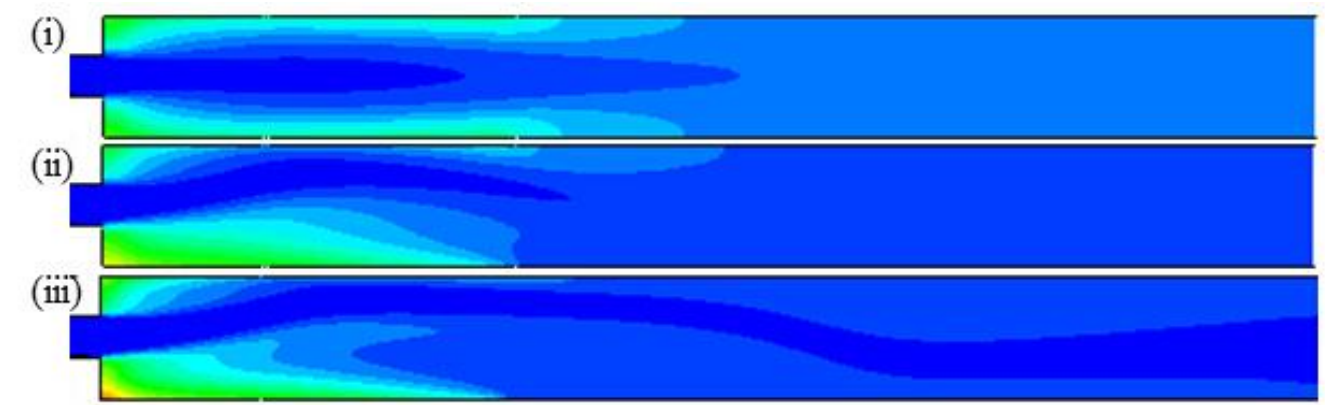

(b)

\title{
(i)
}

\author{
(ii)
}

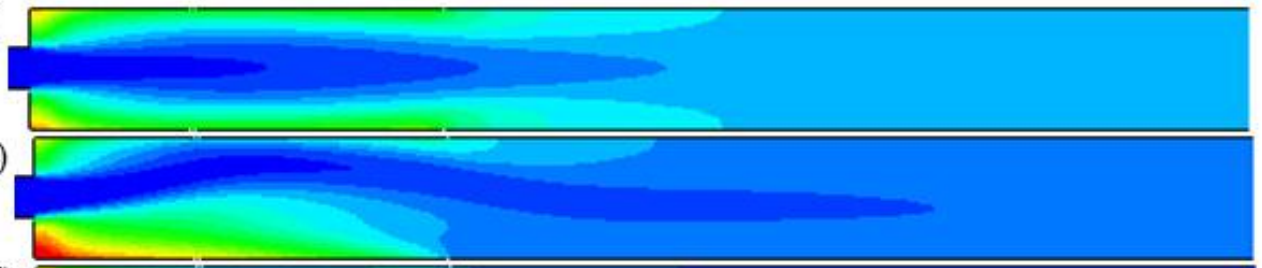

(iii)

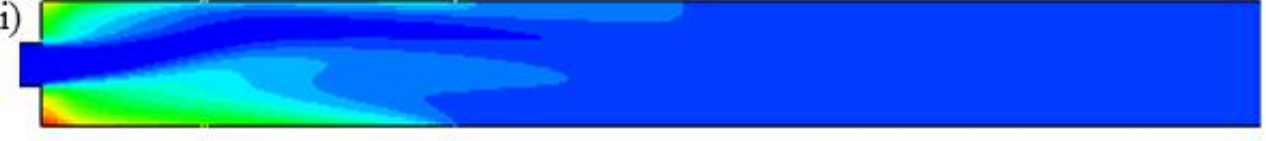

(c)

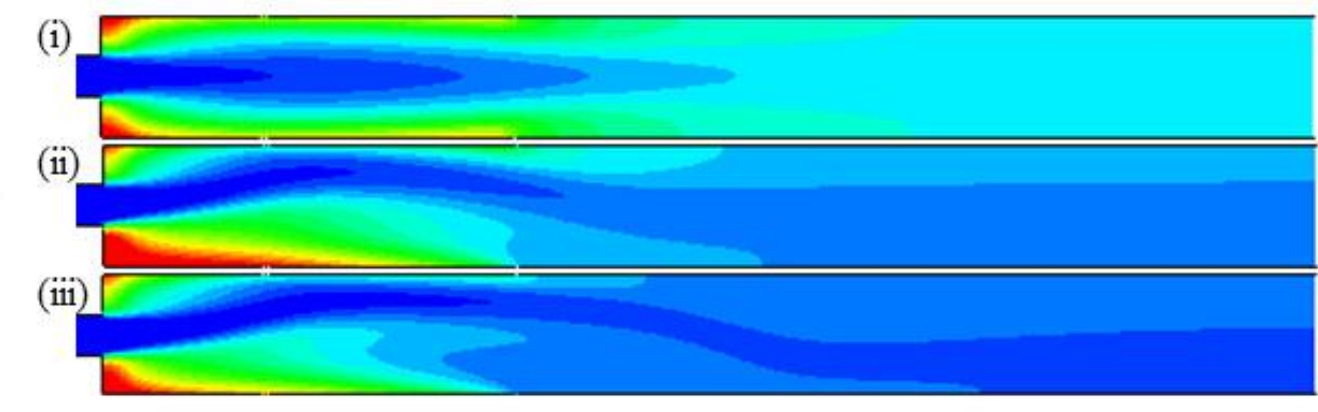

\begin{tabular}{|l|l|}
\hline \multicolumn{1}{|c|}{ Temperature } \\
$317(\mathrm{~K})$ \\
316 \\
315 \\
315 \\
314 \\
313 \\
312 \\
311 \\
310 \\
309 \\
308 \\
307 \\
307 \\
306 \\
305 \\
304 \\
303 \\
302 \\
301 \\
\hline
\end{tabular}

Fig 10 Temperature distributions in the 1:3 planar sudden expansion at three different regimes for each case:

symmetric flow; asymmetric flow; asymmetric flow with a third recirculating eddy at different heat transfer rate values: (a) $q_{w}=100 \mathrm{kw} / \mathrm{m}^{2} ;$ (b) $q_{w}=150 \mathrm{kw} / \mathrm{m}^{2} ;$ (c) $q_{w}=200 \mathrm{kw} / \mathrm{m}^{2} .(\varphi=3 \%)$

The local Nusselt numbers at different heat transfer rates are plotted in Fig. 11. Generally, as the heat transfer rate increases, the local Nusselt number will increase significantly. It is also seen that the rate of heat transfer deterioration at the recirculating area increases with the increasing of the heat transfer rate. Furthermore, it is observed that the peak of the local Nusselt number becomes narrower and sharper as the heat transfer rate increases.

These are because that the decrease of viscosity with temperature changes the local vortex size. The vortex becomes shorter, leading to the advance of the peak. 


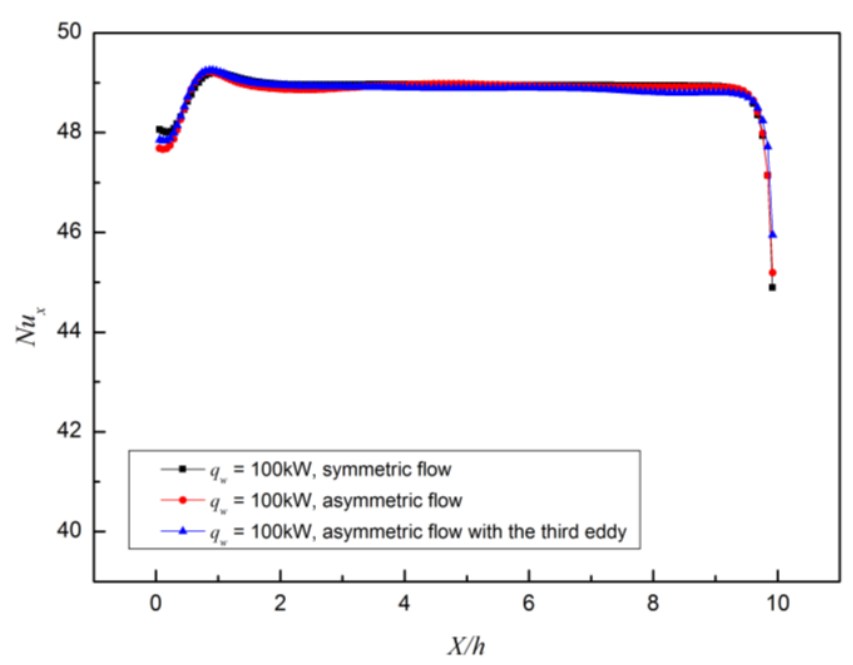

(a)

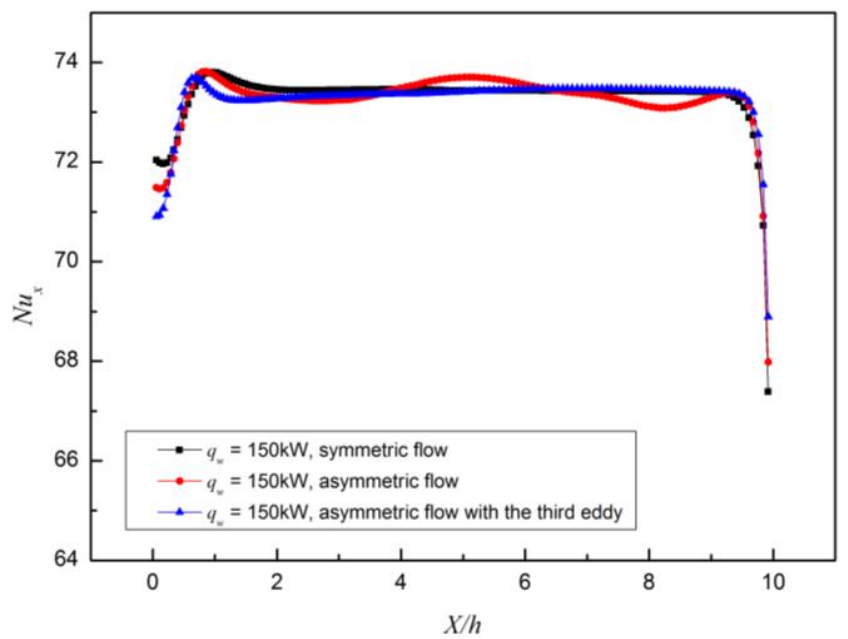

(b)

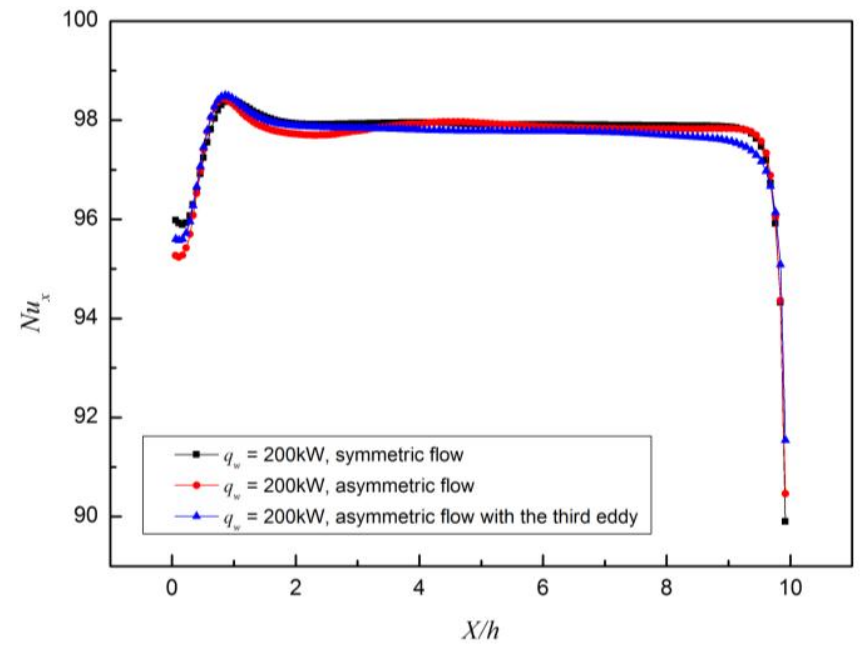

(c)

Fig 11 Local Nusselt number with $x$-axis location at different heat transfer rate: (a) $q_{w}=100 \mathrm{kw} / \mathrm{m}^{2}$; (b) $q_{w}=$ $150 \mathrm{kw} / \mathrm{m}^{2} ;$ (c) $q_{w}=200 \mathrm{kw} / \mathrm{m}^{2} .(\varphi=3 \%)$

\section{Conclusions}

The heat transfer and flow pattern of $\mathrm{Al}_{2} \mathrm{O}_{3}$-water nanofluid in a micro planar sudden expansion have been numerically studied. The nanofluid is treated as a non-Newtonian fluid and the power-law rheology is adopted to describe the characteristics of the flow, in which the flow behavior index depends on the nanoparticle volume 
fraction. We present a systematic study of the $\mathrm{Al}_{2} \mathrm{O}_{3}$-water nanofluid for a wide range of generalized Reynolds number, $50 \leq R e_{\text {gen }} \leq 500$, and nanoparticle volume fraction $0 \% \leq \varphi \leq 3 \%$. The flow is steady for the whole range of $R e_{g e n}$ and the flow strongly depends on the nanoparticle volume fraction as the $R e_{\text {gen }}$ increases. Key points of the performed study are as follows

- Flow bifurcation is delayed for the nanofluids as the nanoparticle volume fraction increases and is advanced as the boundary heat transfer rate increases.

- For nanofluid, the change of the nanoparticle volume fraction affects the fluid rheology and the value of viscosity at the same time, thus the changes of the vortex size with the nanoparticle volume fraction are not monotonous. For the studied range of the nanoparticle volume fraction, the vortex size reaches its maximum value at $2 \%$.

- The heat transfer is enhanced as the nanoparticle volume fraction increases. Especially, the heat transfer deterioration brought by the recirculation area is reduced under a higher nanoparticle volume fraction and the reduction ratio increases with the generalized Reynolds number.

- The boundary heat transfer rate influences the local temperature and thus changes the fluid viscosity, which can slightly change the vortex size and the distribution of the local Nusselt number.

- We also compare the non-Newtonian model results with the results using a Newtonian model. There exists a huge overestimate on the system pressure drop if Newtonian model is taken, and the pressure drop reduction effect becomes more notable when the nanoparticle volume fraction increases.

\section{Acknowledgements}

The support of National Science Foundation of China (No. 51276001) and the common Development Fund of Beijing are gratefully acknowledged. 


\section{Nomenclature}

$C p \quad$ specific heat, $[\mathrm{J} / \mathrm{kg} \mathrm{K}]$

$d_{p} \quad$ nanoparticle diameter, $[\mathrm{m}]$

ER expansion ratio, $H / h$

$h \quad$ width of the upstream channel, [m]

$H \quad$ width of the downstream channel, [m]

$k \quad$ thermal conductivity, [W/m K]

$k_{b} \quad$ Boltzmann constant, $1.3807 \mathrm{e}^{-23}$

$L_{a} \quad$ length of the upstream channel, [m]

$L_{b} \quad$ length of the downstream channel, [m]

$m \quad$ fluid behaviour index, $\left[\mathrm{N} \sec ^{\mathrm{n}} \mathrm{m}^{-2}\right]$

n fluid behaviour index,

$\mathrm{Nu} \quad$ Nusselt number, $h d_{h} / k$

$P \quad$ Pressure, $\left[\mathrm{N} / \mathrm{m}^{2}\right]$

Pe Peclet number, $u_{p} d_{p} / \alpha_{f}$

$q \quad$ heat flux rate, $\left[\mathrm{W} / \mathrm{m}^{2}\right]$

$Q \quad$ heat flux, [W]

$R e_{\text {gen }} \quad$ generalized Reynolds number, $6 \rho u_{i n}{ }^{(2-n)} h_{n} /\{m[(4 n+2) / n] n\}$

$T \quad$ temperature, $[\mathrm{K}]$

$u, v \quad$ velocity component in $\mathrm{x}, \mathrm{y}$-directions, $[\mathrm{m} / \mathrm{s}]$

$u_{p} \quad$ Brownian motion velocity of the particle, $2 k_{b} T /\left(\pi \mu_{f} d_{p}^{2}\right)$ 
Greek symbols

$\begin{array}{ll}\varphi & \text { nanoparticle volume fraction } \\ \rho & \text { density, }\left[\mathrm{kg} / \mathrm{m}^{3}\right] \\ \alpha & \text { thermal diffusion, }\left[\mathrm{N} \mathrm{s} / \mathrm{m}^{2}\right] \\ \gamma & \text { shear rate } \\ \mu & \quad \text { dynamic viscosity, }\left[\mathrm{N} \mathrm{s} / \mathrm{m}^{2}\right] \\ \tau & \\ & \text { shear stress }\end{array}$

\section{Subscripts}

average average value

eff effective

$f \quad$ fluid

heat heating

in inlet

$n f \quad$ nanofluid

$p \quad$ particle

w wall

$x \quad$ X-coordinate

\section{References}

[1] H.A Mohammed, A.A. Al-Aswadi, N.H. Shuaib, R. Saidur, Convective heat transfer and fluid flow study over a 
step using nanofluids: a review, Renew. Sust. Energ. Rev. 15 (2011) 2921-2939.

[2] S. U. S. Choi, Nanofluids: from vision to reality through research, J. Heat Trans. 131 (2009) 1-9.

[3] W. Yu, D. M. France, J. L. Routbort, S. U. S. Choi, Review and comparison of nanofluid thermal conductivity and heat transfer enhancements, Heat Trans. Eng. 29 (2008) 432-460.

[4] S. K. Das, S. U. S. Choi,H. E. Patel, Heat transfer in nanofluids-a review, Heat Trans Eng. 27 (2006) 3-19.

[5] J. Niu, C. Fu, W. Tan, Slip-flow and heat transfer of a non-newtonian nanofluid in a microtube, PLoSONE, 17 (2012) 2-9.

[6] H. Afshar, M. Shams, S.M.M. Nainian, G. Ahmadi, Microchannel heat transfer and dispersion of nanoparticles in slip flow regime with constant heat flux, Int. Commun. Heat Mass Trans. 36 (2009) 1060-1066.

[7] S. Vafaei, D. Wen, Critical heat flux of nanofluids inside a single microchannel: Experiments and correlations, Chem. Eng. Res. Des.92 (2014) 2339-2351.

[8] Mahmoud Ahmed, Morteza Eslamian, Laminar forced convection of a nanofluid in a microchannel: Effect of flow inertia and external forces on heat transfer and fluid flow characteristics, Appl. Therm. Eng. 78 (2015) 326-338.

[9] A.Sh. Kherbeet, H.A. Mohammed, K.M. Munisamy, B.H. Salman, The effect of step height of microscale backward-facing step on mixed convection nano-fluid flow and heat transfer characteristics, Int. J. Heat Mass Trans. 68 (2014) 554-566.

[10] A.Sh. Kherbeet, H.A. Mohammed, K.M. Munisamy, R. Saidur, B.H. Salman, I.M. Mahbubul, Experimental and numerical study of nanofluid flow and heat transfer over microscale forward-facing step, Int. Commun. Heat Mass Trans. 57 (2014) 319-329.

[11] H. Abu-Mulaweh, A review of research on laminar mixed convection flow over backward- and forward- facing steps, Int. J. Therm. Sci. 42 (2003) 897-909. 
[12] M. Sheikholeslami, D. D. Ganji, M. Younus Javed, R. Ellahi, Effect of thermal radiation on magnetohydrodynamics nanofluid flow and heat transfer by means of two phase model, J. Magn. Magn. Mater. 374 (2015) 36-43.

[13] M. Eslamian, M. Ahmed, M.F. El-Dosoky, M.Z. Saghir, Effect of the thermophresis on natural convection in a Rayleigh-Benard cell filled with a nanofluid, Int. J. Heat Mass Trans. 81 (2015) 142-156.

[14] K. Khanafer, K. Vafai, M. Lightstone, Buoyancy-driven heat transfer enhancement in a two-dimensional enclosure utilizing nanofluids, Int. J. Heat Mass Trans. 46 (2003) 3639-3653.

[15] J. Koo, C. Kleinstreuer, Laminar nanofluid flow in micro heat-sinks, Int. J. Heat Mass Trans. 48 (2005) 2652-2661.

[16] P.K. Namburu, D.K. Das, K.M. Tanguturi, R.S. Vajjha, Numerical study of turbulent flow and heat transfer characteristics of nanofluids considering variable properties, Int. J. Therm. Sci. 48 (2009) 290-302.

[17] H. Chang, C.S. Jwo, C.H. Lo, T.T. Tsung, M.J. Kao, Rheology of CuO nanoparticle suspension prepared by ASNSS, Rev. Adv. Mater. Sci. 10 (2004) 128-132.

[18] Y. Ding, H. Alias, D. Wen, R.A. Williams, Heat transfer of aqueous suspensions of carbon nanotubes (CNT nanofluids), Int. J. Heat Mass Trans.49 (2006) 240-250.

[19] A.K. Santra, S. Sen, N. Chakraborty, Study of heat transfer due to laminar flow of copper-water nanofluid through two isothermally heated parallel plates, Int. J. Therm. Sci. 48 (2009) 391-400.

[20] N. Putra, W. Roetzel, S.K. Das, Natural convection of nanofluids, Heat Mass Trans. 39 (2003) 775-784.

[21] W.J. Tseng, C.H. Wu, Aggregation, rheology and electrophoretic packing structure of aqueous $\mathrm{Al}_{2} \mathrm{O}_{3}$ nanoparticle suspensions, Acta. Mater. 50 (2002) 3757-3766.

[22] W.J. Tseng, C.H. Wu, Sedimentation, rheology and particle-packing structure of aqueous $\mathrm{Al}_{2} \mathrm{O}_{3}$ suspensions, Ceram. Int. 29 (2003) 821-828 
[23] B.C. Pak, Y.I. Cho, Hydrodynamic and heat transfer study of dispersed fluids with submicron metallic oxide particles. Exp. Heat Trans. 11 (1998) 151-170.

[24] W.J. Tseng, K.C. Lin, Rheology and colloidal structure of aqueous $\mathrm{TiO}_{2}$ nanoparticle suspensions, Mater. Sci. Eng. A355 (2003) 186-192.

[25] Y. He, Y. Jin, H. Chen, Y. Ding, D. Cang, H. Lu, Heat transfer and flow behaviour of aqueous suspensions of TiO2 nanoparticles (nanofluids) flowing upward through a vertical pipe, Int. J. Heat. Mass. Trans. 50 (2007) $2272-2281$.

[26] J. Chevalier, O. Tillement, F. Ayela, Rheological properties of nanofluids flowing through microchannels, Appl. Phys. Lett. 91 (2007) 233103.

[27] I. Tavman, A. Turgut, M. Chirtoc, H.P. Schuchmann, S. Tavman, Experimental investigation of viscosity and thermal conductivity of suspensions containing nano-sized ceramic particles, Arch. Mater. Sci. Eng. 34 (2008) 99-104.

[28] R. Prasher, D. Song, J. Wang, P. Phelan, Measurements of nanofluid viscosity and its implications for thermal applications, Appl. Phys. Lett. 89 (2006) 133108.

[29] K.B. Anoop, S. Kabelac, T. Sundararajan, S.K. Das, Rheological and flow characteristics of nanofluids: influence of electro viscous effects and particle agglomeration, J. Appl. Phys. 106 (2009) 034909.

[30] GH.R. Kefayati, Simulation of heat transfer and entropy generation of MHD natural convection of non-Newtonian nanofluid in an enclosure, Int. J. Heat Mass Trans. 92 (2016) 1066-1089.

[31]B. Li, Y. Lin, L. Zhu, W. Zhang, Effects of non-newtonian behaviour on the thermal performance of nanofluids in a horizontal channel with discrete regions of heating and cooling, Appl. Therm. Eng. 94 (2016) 404-412.

[32] R. Ellahi, M. Raza, K. Vafai, Series solutions of non-Newtonian nanofluids with Reynolds' model and Vogel's model by means of the homotopy analysis method, Math. Comput. Model. 55 (2012) 1876-1891. 
[33] R. Ellahi, The effects of MHD and temperature dependent viscosity on the flow of non-Newtonian nanofluid in a pipe: Analytical solutions, Appl. Math. Model. 37 (2013) 1451-1467.

[34] N.S. Akbar, S.U. Rahman, R. Ellahi, S. Nadeem, Nano fluid flow in tapering stenosed arteries with permeable walls, Int. J. of Therm. Sci. 85 (2014) 54-61.

[35] S.A. Shehzad, Z. Abdullah, A. Alsaedi, F.M. Abbasi, T. Hayat, Thermally radiative three-dimensional flow of Jeffrey nanofluid with internal heat generation and magnetic field, J. Magn. Magn. Mater. 397 (2016) 108-114.

[36] S.A. Shehzada, Z. Abdullah, F.M. Abbasi, T. Hayat, A. Alsaedi, Magnetic field effect in three-dimensional flow of an Oldroyd-B nanofluid over a radiative surface, Journal of Magnetism and Magnetic Materials 399 (2016) 97-108.

[37] D. Wen, Y. Ding, Experimental investigation into convective heat transfer of nanofluids at the entrance region under laminar flow conditions, Int. J. Heat Mass Trans. 47 (2004) 5181-5188.

[38] H.E. Patel, T. Sundararajan, S.K. Das, An experimental investigation into the thermal conductivity enhancement in oxide and metallic nanofluids, J. Nanoparticle Research 12 (2010) 1015-1031

[39] H.C. Brinkman, The viscosity of concentrated suspensions and solutions, J. Chem. Phys.20 (1952) 571-581.

[40] N. Putra, R. Wilfried, K. Sarit, Natural convection of nanofluids, Heat Mass Transf. 39 (2003) 775-784.

[41] R.J. Poole, B.S. Ridley, Development length requirements for fully-developed laminar pipe flow of inelastic non-Newtonian liquids, ASME J. Fluids Eng. 129 (2007) 1281-1287.

[42] P. Ternik, Planar sudden symmetric expansion flows and bifurcation phenomena of purely viscous shear-thinning fluids, J Non-Newtonian Fluid Mech. 157 (2009) 15-25.

[43] S. Dhinakaran, M.S.N. Oliveira, F.T. Pinho, M.A. Alves, Steady flow of power-law fluids in a 1:3 planar sudden expansion, J. Non-Newtonian Fluid Mech. 198 (2013) 48-58. 